\title{
Scattering amplitudes of Kaluza-Klein strings and extended massive double-copy
}

\author{
Yao Li, ${ }^{a}$ Yan-Feng Hang, ${ }^{a}$ Hong-Jian $\mathbf{H e}^{a, b, c, 1}$ and Song $\mathbf{H e} e^{d, e}$ \\ ${ }^{a}$ Tsung-Dao Lee Institute 85 School of Physics and Astronomy, \\ Key Laboratory for Particle Astrophysics and Cosmology (MOE), \\ Shanghai Key Laboratory for Particle Physics and Cosmology, \\ Shanghai Jiao Tong University, Shanghai 200240, China \\ ${ }^{b}$ Institute of Modern Physics and Department of Physics, \\ Tsinghua University, Beijing 100084, China \\ ${ }^{c}$ Center for High Energy Physics, Peking University, \\ Beijing 100871, China \\ ${ }^{d}$ CAS Key Laboratory of Theoretical Physics, Institute of Theoretical Physics, \\ Chinese Academy of Sciences, Beijing 100190, China \\ ${ }^{e}$ School of Fundamental Physics and Mathematical Sciences, \\ Hangzhou Institute for Advanced Study, UCAS, Hangzhou 310024, China \\ E-mail: neolee@sjtu.edu.cn, yfhang@sjtu.edu.cn, hjhe@sjtu.edu.cn, \\ songhe@itp.ac.cn
}

ABstract: We study the scattering amplitudes of massive Kaluza-Klein (KK) states of open and closed bosonic strings under toroidal compactification. We analyze the structure of vertex operators for the KK strings and derive an extended massive KLT-like relation which connects the $\mathrm{N}$-point KK closed-string amplitude to the products of two KK openstring amplitudes at tree level. Taking the low energy field-theory limit of vanishing Regge slope, we derive double-copy construction formula of the $N$-point massive KK graviton amplitude from the sum of proper products of the corresponding KK gauge boson amplitudes. Then, using the string-based massive double-copy formula, we derive the exact tree-level four-point KK gauge boson amplitudes and KK graviton amplitudes, which fully agree with those given by the KK field-theory calculations. With these, we give an explicit prescription on constructing the exact four-point KK graviton amplitudes from the sum of proper products of the corresponding color-ordered KK gauge boson amplitudes. We further analyze the string-based double-copy construction of five-point and six-point scattering amplitudes of massive KK gauge bosons and KK gravitons.

Keywords: Bosonic Strings, Field Theories in Higher Dimensions, Gauge-gravity correspondence, Scattering Amplitudes

ArXiv EPrint: 2111.12042

\footnotetext{
${ }^{1}$ Corresponding author.
} 


\section{Contents}

1 Introduction 1

2 KK string amplitudes and extended massive KLT-like relation 4

2.1 Compactification of bosonic strings 5

$\begin{array}{lll}2.2 & \text { Vertex operators of KK string states } & 6\end{array}$

2.3 Open and closed string amplitudes for massive KK states 8

$\begin{array}{lll}2.4 & \text { Low energy scattering amplitudes of KK gauge bosons and gravitons } & 10\end{array}$

3 Massive KK open string amplitudes and field theory limit 10

$\begin{array}{lll}3.1 & \text { Elastic amplitudes of KK gauge bosons from KK open strings } & 11\end{array}$

$\begin{array}{lll}3.2 & \text { Inelastic amplitudes of KK gauge bosons from KK open strings } & 13\end{array}$

$\begin{array}{lll}3.3 & \text { Structure of color-ordered massive KK amplitudes } & 15\end{array}$

4 KK graviton amplitudes from extended massive double-copy 18

4.1 Constructing elastic scattering amplitudes of four KK gravitons 18

4.2 Constructing inelastic scattering amplitudes of four KK gravitons 22

4.3 Constructing multi-point scattering amplitudes of KK gravitons 24

5 Conclusions $\quad 25$

A Kinematics of four-point scattering amplitudes of KK states $\quad 27$

B Full scattering amplitudes of KK gauge and Goldstone bosons 28

B.1 Elastic KK gauge and Goldstone boson scattering amplitudes 29

B.2 Inelastic scattering amplitudes of KK gauge and Goldstone bosons 31

B.2.1 Inelastic scattering amplitudes of $(n, n) \rightarrow(m, m) \quad 31$

B.2.2 Inelastic scattering amplitudes of $(0,0) \rightarrow(n, n) \quad 34$

\section{Introduction}

Early attempts of unifying the electromagnetic and gravitational forces pointed to a truly fundamental possibility of a higher dimensional spacetime structure of $5 \mathrm{~d}$ with a single extra spatial dimension compactified on a circle à la Kaluza-Klein (KK) [1,2]. This intriguing avenue was seriously pursued and widely explored in various contexts, including the string/M theories $[3,4]$ and extra dimensional field theories with large or small extra dimensions [5-9]. In fact, the unification among the conventional gauge forces was first realized through the electroweak theory [10-12] of the standard model (SM) and subsequently extended to the grand unification (GUT) of the electroweak and strong forces [13-16]. 
The big obstacle to further unification between the gauge forces and gravity force lies in the apparently distinctive natures of Einstein's generality relativity (GR) including its intricate nonlinearity and perturbative nonrenormalizability. However, the conjectured double-copy relation of GR $=(\text { Gauge Theory })^{2}$ points to fundamental clues to the deep gauge-gravity connection. The Kawai-Lewellen-Tye (KLT) relation [17] was constructed to connect the scattering amplitudes of closed strings to the products of scattering amplitudes of open strings at tree level. In the low energy field-theory limit, the KLT relation leads to the connection of scattering amplitudes of massless gravitons to the products of scattering amplitudes of massless gauge bosons with proper kinematic factors. This was then extended to the field theory framework through the double-copy method of color-kinematics duality of Bern-Carrasco-Johansson (BCJ) [18-20] which links the scattering amplitudes of massless gauge theories to that of the massless gravity. Analyzing the properties of the heterotic string and open string amplitudes can prove and refine parts of the BCJ conjecture [21]. The Cachazo-He-Yuan (CHY) formalism [22-26] shows that the KLT kernel can be interpreted as the inverse amplitudes of bi-adjoint scalars, and this can be generalized to double-copy relations for other field theories $[25,26]$. So far substantial efforts have been made to formulate and test the double-copy constructions between the massless gauge theories and massless GR [20], and some recent works attempted to extend the doublecopy method to the 4d massive Yang-Mills (YM) theory versus Fierz-Pauli-like massive gravity [27-30], to the KK-inspired effective gauge theory with extra global U(1) [31], to compactified 5d KK gauge/gravity theories [32], and to the 3d Chern-Simons theories with or without supersymmetry [33-40].

But the extensions of conventional double-copy method to massive gauge/gravity theories are generally difficult, because many such theories (including the massive YM theory and massive Fierz-Pauli gravity) violate gauge symmetry and diffeomorphism invariance (which are the key for successful double-copy construction). The two important candidates with promise include the compactified KK gauge/gravity theories and the topologically massive Chern-Simons (CS) gauge/gravity theories. The massive KK gauge bosons and KK gravitons acquire their masses from geometric "Higgs" mechanisms [32, 41-43] of the KK compactifications which spontaneously break the higher dimensional gauge symmetry and diffeomorphism invariance to that of $4 \mathrm{~d}$ by boundary conditions. Such geometric "Higgs" mechanisms can be quantitatively formulated at the scattering $S$-matrix level by the KK gauge boson equivalence theorem (KK GAET) [41, 44, 45] and KK gravitational ET (KK GRET) [32], which generally ensure much better high energy behaviors of the KK scattering amplitudes than that of other ill-defined massive theories (with explicitly broken gauge/gravity symmetries) and thus hold real promise for successful double-copy construction. The 3d CS gauge/gravity theories [46, 47] naturally realize topological massgeneration for the gauge bosons (gravitons) in a gauge-invariant (diffeomorphism-invariant) way, which can also ensure good high energy behaviors of the scattering amplitudes [40] and realize successful double-copy constructions [33-40].

A recent work [32] systematically studied the extended BCJ-type double-copy construction between the scattering amplitudes of the massive KK gauge bosons and of the massive KK gravitons in the KK YM gauge theory and KK gravity theory under the $5 \mathrm{~d}$ 
compactification of $\mathbb{S}^{1} / \mathbb{Z}_{2}$. The double-copy construction for the scattering amplitudes of massive KK gauge bosons and KK gravitons is highly nontrivial even for the four-point elastic KK amplitudes due to the presence of double-pole-like structure with exchanges of both zero-modes and KK-modes. Ref. [32] first proposed an improved double-copy method for massive KK amplitudes by using the high energy expansion order by order. The leadingorder (LO) KK gauge boson amplitudes were shown [32] to be mass-independent and their numerators obey the kinematic Jacobi identity at the $O\left(E^{2} M_{n}^{0}\right)$, so the extended BCJ-type double-copy construction can be universally realized to reconstruct the correct LO KK graviton amplitudes. But the next-to-leading-order (NLO) KK gauge boson amplitudes were found [32] to be mass-dependent and the corresponding double-copied KK graviton amplitudes do not always match the exact KK graviton amplitudes at the NLO. So the naive extension of the BCJ double-copy method could not fully work and a modified massive double-copy construction was proposed [32] for the NLO KK amplitudes, ${ }^{1}$ but this is yet to be established for all KK scattering processes and for going beyond the NLO. Hence, it is truly attractive and important to establish the double-copy construction, from the first principle of KK string theory formulation, for the exact tree-level massive KK gauge-boson/graviton amplitudes and in a universal way.

In this work, we take the simplest KK compactification of the $26 \mathrm{~d}$ bosonic string theory $[3,4]$ as a tool to derive the extended massive KLT-like relations for KK closed/openstring amplitudes and then achieve the doubel-copy construction of the realistic $5 \mathrm{~d}$ KK gauge boson/graviton amplitudes in the low energy field-theory limit. The essential advantage of the compactified KK string theory is that the connection between the KK closedstring amplitudes and the proper products of KK open-string amplitudes can be intrinsically built in from the start. A recent literature [48] studied the general KLT factorization of winding string amplitudes in the bosonic string theory and computed explicitly the fourpoint tachyon amplitudes, but did not consider the amplitudes in the low energy field-theory limit. We will study the scattering amplitudes of massive KK states of open and closed bosonic strings, and derive the corresponding scattering amplitudes of KK gauge-bosons and of KK gravitons in the field-theory limit. With these, we derive the extended KLT-like relations which connect the $N$-point KK closed-string amplitude to the product of the two corresponding open string amplitudes at tree level. Taking the field-theory limit of vanishing Regge slope, we derive double-copy construction which formulates the general $N$-point massive KK graviton amplitude as the sum of proper products of the corresponding KK gauge boson amplitudes. Then, using the string-based KLT-like massive KK double-copy formula, we derive the exact four-point elastic and inelastic scattering amplitudes of KK gravitons from the sum of the proper products of the relevant color-ordered amplitudes of KK gauge bosons at tree level. We verify that the reconstructed four-point elastic KK graviton amplitude fully agrees with that given by the available Feynman-diagram calculations $[49,50]$ in the $5 \mathrm{~d}$ KK field theory of GR. Based on our KK-string formulation, we give an explicit prescription on constructing the exact four-point KK graviton scattering

\footnotetext{
${ }^{1}$ As we will show in appendix B of the present paper, we can construct a new type of numerators by making generalized gauge transformations and properly choosing the energy expansion parameter such that the kinematic Jacobi identity is obeyed. But further improvements are still needed.
} 
amplitudes from the sum of relevant products of the corresponding color-ordered KK gauge boson scattering amplitudes. We further analyze the string-based double-copy construction of five-point and six-point scattering amplitudes of massive KK gauge bosons and KK gravitons.

This paper is organized as follows. In section 2, we analyze the structure of vertex operators for the KK strings and derive an extended massive KLT-like relation which connects the $\mathrm{N}$-point $\mathrm{KK}$ closed-string amplitude to the product of the two corresponding open-string amplitudes at tree level. Then, we take the low energy field-theory limit of vanishing Regge slope and present the double-copy construction of the $N$-point massive KK graviton amplitude by the sum of relevant products of the color-ordered KK gauge boson amplitudes. In section 3, we systematically derive the four-point elastic and inelastic color-ordered scattering amplitudes of KK gauge bosons from the field-theory limit of the corresponding scattering amplitudes of KK open strings. We study the structure of these color-ordered massive KK gauge boson amplitudes and demonstrate that they can be obtained from the scattering amplitudes of massless zero-mode gauge bosons under proper shifts of the Mandelstam variables. This gives an elegant and efficient way to compute any color-ordered massive KK gauge boson amplitudes. In section 4, applying the string-based massive double-copy formula, we derive the exact tree-level four-point elastic and inelastic KK graviton amplitudes. Then, we give an explicit prescription on constructing the exact four-point KK graviton amplitudes from the sum of relevant products of the corresponding color-ordered KK gauge boson amplitudes. We also use our general string-based doublecopy construction to obtain the five-point and six-point scattering amplitudes of massive KK gauge bosons and KK gravitons. Finally, we conclude in section 5. Appendix A provides the notational setup and kinematics formulas for the elastic and inelastic scattering processes of four KK states. In appendix B we present the exact four-point amplitudes of the elastic and inelastic scattering of KK gauge bosons at tree level, which are shown to fully agree with those obtained from the corresponding scattering amplitudes of KK open strings under low energy field-theory limit as given by section 3. This also serves as a systematic consistency check of our open-string calculations in the main text.

\section{KK string amplitudes and extended massive KLT-like relation}

In this section, we consider compactifications of the $26 \mathrm{~d}$ bosonic string theory with one relatively large extra spatial dimension compactified to a circle and with all other extra spatial dimensions decoupled due to their extremely small radii of $\mathcal{O}\left(M_{\mathrm{Pl}}^{-1}\right)$. We study the mass spectra of both KK open and closed strings. Then, we explicitly compute the $N$-point KK open-string amplitudes under compactification by using the relevant compact photon vertex operators and construct the amplitudes of KK closed-strings by products of two KK open-string amplitudes. The scattering amplitudes of KK closed-strings take the KLT-like form. Finally, taking the low energy field-theory limit $\alpha^{\prime} \rightarrow 0$, we derive the formulas of general $N$-point amplitudes in the compactified KK field theories, so the KLT-like relation of KK string amplitudes will result in the double-copy formula of the corresponding KK graviton amplitudes. 


\subsection{Compactification of bosonic strings}

For the sake of the present study, we consider the bosonic strings propagating in a 26dimensional spacetime background $\mathbb{R}^{1,24} \times \mathbb{S}^{1}$. We can first compactify the extra spatial dimensions of coordinates $\left\{X^{4}, \cdots, X^{24}\right\}$ with their radii $r_{j}=\mathcal{O}\left(M_{\mathrm{Pl}}^{-1}\right) \ll R(j=4, \cdots, 24)$, so they are fully decoupled at energy scales much below the reduced Planck scale $M_{\mathrm{Pl}}=$ $(8 \pi G)^{-1 / 2}$. Thus, we only need to study the toroidal compactification of the single extra spatial dimension of coordinate $X^{25}$ on a circle $\mathbb{S}^{1}$ with radius $R$, which is much larger than the Planck length $M_{\mathrm{Pl}}^{-1}$ and does not decouple in our low energy effective theory. (Here by low energy, we mean the energy scale scales which are lower than the reduced Planck scale $M_{\mathrm{Pl}}$ by about two orders of magnitude or more.) For the present study, we take bosonic string as a computational tool for establishing the massive KLT-like relations of KK string states and for deriving the low energy KK graviton scattering amplitudes.

Then, we can identify the coordinate $X^{25}(\equiv X)$ as a scalar field on the string worldsheet and it obeys the periodic boundary condition on the circle $\mathbb{S}^{1}$ :

$$
X \cong X+2 \pi R
$$

For the closed strings, without loss of generality, the periodic boundary condition further takes the following form:

$$
X(\tau, \sigma+2 \pi)=X(\tau, \sigma)+2 \pi w R,
$$

where $w \in \mathbb{Z}$ is the winding number describing how many times a closed string winds around the circle $\mathbb{S}^{1}$. Thus, the eigenvalues of generators of Virasoro algebra $\left(L_{0}, \bar{L}_{0}\right)$ for the oscillation modes of closed string are derived as follows:

$$
\begin{aligned}
& L_{0}=\frac{\alpha^{\prime}}{4} p^{\mu} p_{\mu}+\frac{\alpha^{\prime}}{4}\left(\frac{\hat{n}}{R}+\frac{w R}{\alpha^{\prime}}\right)^{2}+(N-1), \\
& \bar{L}_{0}=\frac{\alpha^{\prime}}{4} p^{\mu} p_{\mu}+\frac{\alpha^{\prime}}{4}\left(\frac{\hat{n}}{R}-\frac{w R}{\alpha^{\prime}}\right)^{2}+(\tilde{N}-1),
\end{aligned}
$$

where $\alpha^{\prime}$ is the Regge slope, $\hat{n} \in \mathbb{Z}$ denotes the Kaluza-Klein (KK) level, and $(N, \tilde{N})$ represent the string level. Hence, the mass spectrum of the KK state of a closed string can be obtained by imposing the physical conditions $L_{0}=\bar{L}_{0}=0$ :

$$
M_{\mathrm{cl}}^{2}=\left(p_{\hat{n}}+\frac{w R}{\alpha^{\prime}}\right)^{2}+\frac{4}{\alpha^{\prime}}(N-1)=\left(p_{\hat{n}}-\frac{w R}{\alpha^{\prime}}\right)^{2}+\frac{4}{\alpha^{\prime}}(\tilde{N}-1),
$$

where

$$
p_{\hat{n}}=\frac{\hat{n}}{R}=\operatorname{sgn} \times M_{n},
$$

with $\hat{n} \in \mathbb{Z}$ and $\operatorname{sgn} \equiv \operatorname{sign}(\hat{n})= \pm 1$. In the above, $M_{n}=|\hat{n}| / R$ is the KK mass-parameter. Note that in eq. (2.4), the second equality is realized by imposing the level matching condition $n w=\widetilde{N}-N$. We can further decompose the mass spectrum (2.4) as follows:

$$
M_{\mathrm{cl}}^{2}=M_{n}^{2}+\frac{w^{2} R^{2}}{\alpha^{\prime 2}}+\frac{2}{\alpha^{\prime}}(N+\widetilde{N}-2),
$$


where the right-hand-side (r.h.s.) contains the contributions from the squared KK-mass $M_{n}^{2}=n^{2} / R^{2}$ and a squared topological mass $\left(w R / \alpha^{\prime}\right)^{2}$ (related to the string winding number). For studying the low energy limit of string theory as a field theory, we will set $w=0$ and thus $N=\widetilde{N}=1$ for the (KK) gravitons.

The open string satisfies the Dirichlet boundary condition for the compactified dimensions and the Neumann boundary condition for non-compactified dimensions. Each ending point of the open string is attached to a D-brane $[3,4]$. The boundary condition for the open string takes the following form:

$$
X(\tau, \sigma+\pi)=X(\tau, \sigma)+2 \pi w R+q L,
$$

where $q \in \mathbb{Z}$ labels the D-branes transverse to the compactified circle $\mathbb{S}^{1}$ with equal distance $L$. We make the following mode expansion for open string:

$$
X(\tau, \sigma)=x_{q}+\frac{\sigma}{\pi}\left(q^{\prime} L+2 \pi w R\right)-\sqrt{2 \alpha^{\prime}} \sum_{m \neq 0} \frac{\alpha_{m}}{m} e^{-\mathrm{i} m \tau} \sin (m \sigma),
$$

where we set the two ends of the open string at the $q$-th brane and $\left(q+q^{\prime}\right)$-th brane:

$$
X(\tau, 0)=x_{q}, \quad X(\tau, \pi)=x_{q}+q^{\prime} L .
$$

Thus, the mass spectrum of open strings is derived as follows:

$$
M_{\mathrm{op}}^{2}=\left(\frac{\hat{n} L+2 \pi w R}{2 \pi \alpha^{\prime}}\right)^{2}+\frac{1}{\alpha^{\prime}}(N-1)
$$

For studying the low energy limit of string theory as a field theory, we only need to consider the $w=0$ case and thus $N=1$ for the (KK) gauge bosons. The mass spectra (2.4) and (2.10) are not necessarily identical in general. But, for the consistent realization of doublecopy construction, we can impose the following matching condition on eq. (2.10):

$$
R L=2 \pi \alpha^{\prime},
$$

and make the rescaling $\left(\alpha^{\prime}, R, L\right) \rightarrow \frac{1}{4}\left(\alpha^{\prime}, R, L\right)$, such that the mass spectrum (2.10) of open strings coincides with the mass spectrum (2.4) of closed strings [48].

\section{$2.2 \quad$ Vertex operators of KK string states}

In this subsection, we present the vertex operators for the KK string states. For the closed and open strings under compactification, we can write down the integrated vertex operators for their KK states:

$$
\begin{aligned}
V_{\text {op }}(\zeta, k, \hat{n}) & =\mathrm{i} g_{\text {op }} \int \mathrm{d} y \zeta_{\mu}: \partial X^{\mu} e^{\mathrm{i} k \cdot X} e^{\mathrm{i} p_{\hat{n}} \mathcal{Y}}: \\
V_{\mathrm{cl}}(\zeta, k, \hat{n}) & =\mathrm{i} g_{\mathrm{cl}} \int \mathrm{d}^{2} z \zeta_{\mu \nu}: \partial X^{\mu} \bar{\partial} X^{\nu} e^{\mathrm{i} k \cdot X} e^{\mathrm{i} p_{\hat{n}} \mathcal{X}}:,
\end{aligned}
$$

where $k$ denotes the momentum in the noncompactified spacetime and $p_{\hat{n}}=\hat{n} / R(\hat{n} \in \mathbb{Z})$ is the quantized momentum in $26 \mathrm{~d}$. The compactified $26 \mathrm{~d}$ string coordinates $(\mathcal{X}, \mathcal{Y})$ are defined as:

$$
\mathcal{Y}(y)=X_{L}(y)-X_{R}(y), \quad \mathcal{X}(z, \bar{z})=X_{L}(z)+X_{R}(\bar{z})
$$


where $X_{L}(z)$ and $X_{R}(\bar{z})$ denote the left-moving and right-moving string coordinates in the $26 \mathrm{~d}$, respectively.

Then, for a noncompactified spatial dimension, we can write down the Green functions for the open strings under the Neumann boundary condition:

$$
\begin{aligned}
& \left\langle X_{L}^{\mu}\left(y_{1}\right) X_{R}^{\nu}\left(y_{2}\right)\right\rangle=\left\langle X_{R}^{\mu}\left(y_{1}\right) X_{L}^{\nu}\left(y_{2}\right)\right\rangle=0, \\
& \left\langle X_{L}^{\mu}\left(y_{1}\right) X_{L}^{\nu}\left(y_{2}\right)\right\rangle=\left\langle X_{R}^{\mu}\left(y_{1}\right) X_{R}^{\nu}\left(y_{2}\right)\right\rangle=-\alpha^{\prime} \eta^{\mu \nu} \ln \left|y_{1}-y_{2}\right|,
\end{aligned}
$$

where the Lorentz indices $\mu, \nu=0,1, \ldots, 24$. While for a compactified spatial dimension, we have the Green functions of open strings under the Dirichlet boundary condition:

$$
\begin{aligned}
& \left\langle X_{L}\left(y_{1}\right) X_{R}\left(y_{2}\right)\right\rangle=\left\langle X_{R}\left(y_{1}\right) X_{L}\left(y_{2}\right)\right\rangle=\alpha^{\prime} \ln \left|y_{1}-y_{2}\right|, \\
& \left\langle X_{L}\left(y_{1}\right) X_{L}\left(y_{2}\right)\right\rangle=\left\langle X_{R}\left(y_{1}\right) X_{R}\left(y_{2}\right)\right\rangle=-\alpha^{\prime} \ln \left|y_{1}-y_{2}\right| .
\end{aligned}
$$

And the Green functions for closed string is given by

$$
\left\langle X^{M}\left(z_{1}, \bar{z}_{1}\right) X^{N}\left(z_{2}, \bar{z}_{2}\right)\right\rangle=-\frac{\alpha^{\prime}}{2} \eta^{M N} \ln \left(\left|z_{1}-z_{2}\right|^{2}\right),
$$

where the $26 \mathrm{~d}$ Lorentz indices $M, N=(0,1, \ldots, 25)$.

In string theory, imposing the orbifold compactification $\mathbb{S}^{1} / \mathbb{Z}_{2}$ will generate an antiperiodic boundary condition, which lifts the vacuum energy on the worldsheet and modifies the mass spectrum [3, 4]. In consequence, the mass of each KK open-string state gets a shift $\Delta M_{\mathrm{op}}^{2}=\frac{1}{16 \alpha^{\prime}}$, and the mass of each KK closed-string state receives an increase $\Delta M_{\mathrm{cl}}^{2}=\frac{1}{4 \alpha^{\prime}}$. Thus they will be decoupled in the field-theory limit of $\alpha^{\prime} \rightarrow 0$. Hence, we will first make our analysis by using the periodic boundary condition on $\mathbb{S}^{1}$ without imposing the $\mathbb{Z}_{2}$. Then, we can construct the vertex operators for KK open strings and KK closed strings with specified $\mathbb{Z}_{2}$ parity:

$$
\begin{aligned}
& V_{\mathrm{op}}^{ \pm}(\zeta, k, n)=\frac{1}{\sqrt{2}}\left[V_{\mathrm{op}}(\zeta, k,+n) \pm V_{\mathrm{op}}(\zeta, k,-n)\right], \\
& V_{\mathrm{cl}}^{ \pm}(\zeta, k, n)=\frac{1}{\sqrt{2}}\left[V_{\mathrm{cl}}(\zeta, k,+n) \pm V_{\mathrm{cl}}(\zeta, k,-n)\right],
\end{aligned}
$$

where $n \in \mathbb{Z}^{+}$and the superscript " $+(-)$" stands for the $\mathbb{Z}_{2}$-even $\left(\mathbb{Z}_{2}\right.$-odd) state. We note that for the zero-modes $(n=0)$, vertex operators for open and closed strings, $V_{\mathrm{op}}(\zeta, k, 0)$ and $V_{\mathrm{cl}}(\zeta, k, 0)$, are always $\mathbb{Z}_{2}$-even. In particular, we will be interested in the above vertex operators having $\mathbb{Z}_{2}$-even parity because their scattering amplitudes will give, in the low energy field-theory limit, the corresponding scattering amplitudes of the KK gauge bosons and of the KK gravitons in the KK gauge/gravity theories under the 5d compactification of $\mathbb{S}^{1} / \mathbb{Z}_{2}$. This will also be valuable for comparison with the literature $[32,41,50]$ which computed some of these KK amplitudes by Feynman diagram approach in the KK gauge/gravity field theories under $5 \mathrm{~d}$ compactification of $\mathbb{S}^{1} / \mathbb{Z}_{2}$. 


\subsection{Open and closed string amplitudes for massive KK states}

For a $N$-point KK string amplitude, the conservation of the compactified momentum (KKnumber) is achieved by the neutral condition [53] of the vertex operators on the string worldsheet, i.e., the sum of KK numbers $\left(\hat{n}_{j}\right)$ of the external states should vanish:

$$
\sum_{j=1}^{N} \hat{n}_{j}=\sum_{j=1}^{N} \operatorname{sgn}_{j} \times n_{j}=0,
$$

where $\hat{n}_{j} \in \mathbb{Z}$ is the KK number of the $j$-th external state, $n_{j}=\left|\hat{n}_{j}\right| \in \mathbb{Z}^{+}$, and the sign of $\hat{n}_{j}$ is denoted as $\operatorname{sgn}_{j}=\operatorname{sign}\left(\hat{n}_{j}\right)$. Thus we can write $p_{\hat{n}_{j}}=\operatorname{sgn}_{j} \times M_{n_{j}}$. Hence, the tree-level $N$-point open-string amplitude can be written as follows:

$$
\begin{aligned}
& \mathcal{A}_{\mathrm{op}}^{(N)}(\zeta, k, n) \\
& \quad=\frac{e^{-\lambda}}{\operatorname{Vol}[\mathrm{SL}(2, \mathbb{R})]} \prod_{j=1}^{N} \int \mathrm{d} y_{j}\left\langle V_{\mathrm{op}}^{ \pm}\left(\zeta_{1}, k_{1}, n_{1}\right) \cdots V_{\mathrm{op}}^{ \pm}\left(\zeta_{N}, k_{N}, n_{N}\right)\right\rangle \\
& \quad=\frac{(-)^{\omega} 2^{-\widehat{N} / 2} e^{-\lambda}}{\operatorname{Vol}[\mathrm{SL}(2, \mathbb{R})]} \prod_{j=1}^{N} \sum_{\left\{\operatorname{sgn}_{j}\right\}} \int \mathrm{d} y_{j}\left\langle V_{\mathrm{op}}\left(\zeta_{1}, k_{1}, \operatorname{sgn}_{1} n_{1}\right) \cdots V_{\mathrm{op}}\left(\zeta_{N}, k_{N}, \operatorname{sgn}_{N} n_{N}\right)\right\rangle,
\end{aligned}
$$

where $\lambda$ denotes the vacuum expectation value of the dilaton and in the second line we have further expressed the amplitude in terms of open-string vertex operators from the r.h.s. of eq. (2.17a). Here we choose each external state to be $\mathbb{Z}_{2}$ even (odd), corresponding to its vertex operator $V_{\text {op }}^{ \pm}\left(\zeta_{j}, k_{j}, n_{j}\right)$ being $\mathbb{Z}_{2}$ even (odd) as indicated by its superscript $+(-)$. [An external state of the amplitude $\mathcal{A}_{\mathrm{op}}^{(N)}(\zeta, k, n)$ can also be chosen as non-eigenstate of $\mathbb{Z}_{2}$ parity and thus its corresponding vertex operator is $V_{\mathrm{op}}\left(\zeta_{j}, k_{j}, \pm n_{j}\right)$. Such cases can be studied by our formulation as well, although our present study will focus on the $N$-point amplitude like eq. (2.19).] In the second line of eq. (2.19), the overall coefficient contains the sign factor $(-)^{\omega}$, where $\omega=0$ for all vertex operators being $\mathbb{Z}_{2}$-even and $\omega \neq 0$

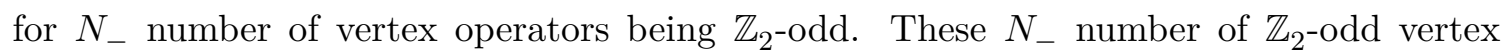
operators will contribute a nontrivial sign factor $(-)^{\omega}$, where $\omega=\sum_{j=1}^{N_{-}}\left[1+H\left(\operatorname{sgn}_{j}\right)\right]$ and the Heaviside step function $H(+)=1$ and $H(-)=0$. In eq. (2.19), we use $\widehat{N}$ to denote the number of the external KK excitation states (with $n_{j}>0$ ) and the factor $2^{-\widehat{N} / 2}$ arises from the overall coefficient $1 / \sqrt{2}$ of eq. (2.17a). So the number of possible external zero-mode states equals $(N-\widehat{N})$. In the second line of eq. (2.19), the summation over $\left\{\operatorname{sgn}_{j}\right\}$ means to sum up all allowed sign-combinations of KK numbers of external states which obey the condition (2.18). Integrating over $y_{j}$, we can reexpress the $N$-point $\mathrm{KK}$ open string amplitude as follows:

$$
\mathcal{A}_{\mathrm{op}}^{(N)}(\zeta, k)=\left(\frac{1}{2}\right)^{\frac{\widehat{N}}{2}} \sum_{\left\{\operatorname{sgn}_{j}\right\}} \sum_{\alpha \in S_{N-1}} \mathcal{A}_{\mathrm{op}}^{(N)}\left[\zeta_{j}, \hat{k}_{j} \mid\{1, \alpha(2 \cdots N)\}\right] \times T[1, \alpha(2 \cdots N)],
$$

where $\hat{k}_{j}=\left(k_{j}^{\mu}, \operatorname{sgn}_{j} \times M_{n_{j}}\right)$ is the $26 \mathrm{~d}$ momentum and the notation $T[1, \alpha(2 \cdots N)]=$ $\operatorname{Tr}\left(T^{1} T^{\alpha(2)} \cdots T^{\alpha(N)}\right)$ denotes the Chan-Paton factor. The partial amplitudes on the r.h.s. 
of eq. (2.20) are not fully independent, among which only $(N-3)$ ! partial amplitudes are independent [18-20]. We further express the color-ordered partial amplitude on the r.h.s. of eq. (2.20) as follows:

$$
\mathcal{A}_{\mathrm{op}}^{(N)}\left[\zeta_{j}, \hat{k}_{j} \mid\{1, \alpha(2 \cdots N)\}\right]=\mathrm{i} g_{\mathrm{op}}^{N} C_{D_{2}}(2 \pi)^{26} \delta^{(26)}\left(\sum_{j} \hat{k}_{j}\right) \overline{\mathcal{A}}_{\mathrm{op}}^{(N)}\left[\zeta_{j}, \hat{k}_{j} \mid\{1, \alpha(2 \cdots N)\}\right],
$$

where the coefficient $C_{D_{2}}$ is given by $C_{D_{2}}=e^{-\lambda}\left(C_{D_{2}}^{g} C_{D_{2}}^{X}\right)$. The constants $C_{D_{2}}^{g}$ and $C_{D_{2}}^{X}$ are given by the path integral of the zero mode of the $b c$ ghost and $X$ scalar field on the string worldsheet, respectively. We will further compute the reduced amplitude $\overline{\mathcal{A}}_{\mathrm{op}}^{(N)}$ explicitly for the 4-point scattering in section 3.

The closed-string amplitude can be derived from the product of two open-string amplitudes [17]. Using the open-string amplitude (2.20), we construct the $N$-point massive KK closed-string amplitude at tree level:

$$
\begin{aligned}
\mathcal{A}_{\mathrm{cl}}^{(N)}(\zeta, k)= & \mathrm{i}(2 \pi)^{26} \delta^{(26)}\left(\sum_{j} \hat{k}_{j}\right) \overline{\mathcal{A}}_{\mathrm{cl}}^{(N)}(\zeta, k), \\
\overline{\mathcal{A}}_{\mathrm{cl}}^{(N)}(\zeta, k)= & g_{\mathrm{cl}}^{N} C_{S_{2}}\left(\frac{1}{2}\right)^{\widehat{N} / 2}\left(-\frac{\pi \alpha^{\prime}}{2}\right)^{N-3} \sum_{\left\{a_{j}, b_{j}\right\}\left\{\mathrm{sgn}_{j}\right\}\{\alpha, \beta\} \in S_{N-3}}\left\{\widehat{\varrho}_{a b} \mathcal{S}_{\mathrm{ST}}^{\alpha^{\prime}}[\alpha \mid \beta]_{\hat{k}_{1}}\right. \\
& \times \overline{\mathcal{A}}_{\mathrm{op}}^{(N)}\left[\zeta_{j}^{a_{j}}, \hat{k}_{j} \mid\{1, \alpha(2 \cdots N-2), N-1, N\}\right] \\
& \left.\times \overline{\mathcal{A}}_{\mathrm{op}}^{(N)}\left[\zeta_{j}^{b_{j}}, \hat{k}_{j} \mid\{N-1, N, \beta(2 \cdots N-2), 1\}\right]\right\}\left.\right|_{\alpha^{\prime} \rightarrow \alpha^{\prime} / 4},
\end{aligned}
$$

where $C_{S_{2}}=e^{-2 \lambda}\left(C_{S_{2}}^{g} C_{S_{2}}^{X}\right)=32 \pi /\left(\alpha^{\prime 3} g_{\mathrm{cl}}^{2}\right)$. In the above eq. $(2.22 \mathrm{~b})$, the polarization tensor $\zeta_{\mu \nu}$ of closed strings is expressed as a sum of the products of polarization vectors of two open strings:

$$
\zeta_{\mu \nu}=\varrho_{a b} \zeta_{\mu}^{a} \zeta_{\nu}^{b}
$$

where the coefficient $\varrho_{a b} \in \mathbb{R}$. In eq. $(2.22 \mathrm{~b})$, the coefficient $\widehat{\varrho}_{a_{j} b_{j}}$ is defined as the product of $\varrho_{a b}$ for all external graviton states:

$$
\widehat{\varrho}_{a b}=\prod_{j=1}^{N} \varrho_{a_{j} b_{j}}
$$

The string momentum kernel $\mathcal{S}_{\mathrm{ST}}^{\alpha^{\prime}}[\alpha \mid \beta]_{\hat{k}_{1}}$ connects the two open-string amplitudes and takes the following explicit form [52]:

$$
\mathcal{S}_{\mathrm{ST}}^{\alpha^{\prime}}\left[\alpha_{1} \cdots \alpha_{j} \mid \beta_{1} \cdots \beta_{j}\right]_{\hat{k}_{1}}=\left(\frac{\pi \alpha^{\prime}}{2}\right)^{-j} \prod_{i=1}^{j} \sin \left[\pi \alpha^{\prime}\left(\hat{k}_{1} \cdot \hat{k}_{\alpha_{i}}+\sum_{\ell>i}^{j} \Theta\left(\alpha_{i}, \alpha_{\ell}\right) \hat{k}_{\alpha_{i}} \cdot \hat{k}_{\alpha_{\ell}}\right)\right],
$$

where the step function $\Theta\left(\alpha_{i}, \alpha_{\ell}\right)=1$ when the order of $\left(\alpha_{i}, \alpha_{\ell}\right)$ is opposite in $\left(\left\{\alpha_{i}\right\},\left\{\beta_{i}\right\}\right)$, while $\Theta\left(\alpha_{i}, \alpha_{\ell}\right)=0$ when the order of $\left(\alpha_{i}, \alpha_{\ell}\right)$ is the same as in $\left(\left\{\alpha_{i}\right\},\left\{\beta_{i}\right\}\right)$. 


\subsection{Low energy scattering amplitudes of KK gauge bosons and gravitons}

In this subsection, we will derive the extended massive KLT-like relations of KK states for the low energy field theory. For this purpose, we take the limit of zero Regge slop $\alpha^{\prime} \rightarrow 0$ for the closed-string amplitude in eqs. (2.22) and (2.25). Then, the open/closed-string amplitudes and the string momentum kernel will reduce to their corresponding field-theory expressions:

$$
\mathcal{A}_{\mathrm{op}} \rightarrow \mathcal{T}, \quad \mathcal{A}_{\mathrm{cl}} \rightarrow \mathcal{M}, \quad \mathcal{S}_{\mathrm{ST}}^{\alpha^{\prime}} \rightarrow \mathcal{S}_{\mathrm{FT}}
$$

With these, we can derive the following low energy $N$-point graviton scattering amplitude:

$$
\begin{aligned}
\mathcal{M}^{(N)}(\zeta, k)= & \left(\frac{\kappa}{4}\right)^{N-2} 2^{-\widehat{N} / 2}(-1)^{N+1} \sum_{\left\{a_{j}, b_{j}\right\}} \sum_{\left\{\operatorname{sgn}_{j}\right\}\{\alpha, \beta\} \in S_{N-3}}\left\{\widehat{\varrho}_{a b} \mathcal{S}_{\mathrm{FT}}[\alpha \mid \beta]_{\hat{k}_{1}}\right. \\
& \times \mathcal{T}^{(N)}\left[\zeta_{j}^{a_{j}}, \hat{k}_{j} \mid\{1, \alpha(2 \cdots N-2), N-1, N\}\right] \\
& \left.\times \mathcal{T}^{(N)}\left[\zeta_{j}^{b_{j}}, \hat{k}_{j} \mid\{N-1, N, \beta(2 \cdots N-2), 1\}\right]\right\},
\end{aligned}
$$

where the gravitational coupling $\kappa$ and the closed-string coupling $g_{\mathrm{cl}}$ are connected by the relation $\kappa=2 \pi \alpha^{\prime} g_{\mathrm{cl}}$. In the above, $\mathcal{S}_{\mathrm{FT}}[\alpha \mid \beta]_{\hat{k}_{1}}$ is the momentum kernel in the field theory limit and takes the following form $[51,52]$ :

$$
\mathcal{S}_{\mathrm{FT}}\left[\alpha_{1} \cdots \alpha_{j} \mid \beta_{1} \cdots \beta_{j}\right]_{\hat{k}_{1}}=\prod_{i=1}^{j}\left(2 \hat{k}_{1} \cdot \hat{k}_{\alpha_{i}}+2 \sum_{\ell>i}^{j} \Theta\left(\alpha_{i}, \alpha_{\ell}\right) \hat{k}_{\alpha_{i}} \cdot \hat{k}_{\alpha_{\ell}}\right) .
$$

\section{Massive KK open string amplitudes and field theory limit}

In this section, we compute explicitly the four-point color-ordered elastic and inelastic scattering amplitudes of KK open strings and derive the corresponding KK gauge boson scattering amplitudes in the low energy field-theory limit.

Thus, we compute the four-point color-ordered partial amplitudes of KK open-string scattering with three fixed points $\left(y_{1}, y_{2}, y_{3}\right)=(0,1, \infty)$ :

$$
\begin{aligned}
& g_{\mathrm{op}}^{4} C_{D_{2}} \overline{\mathcal{A}}_{\mathrm{op}}[1243]=\frac{g_{\mathrm{op}}^{2}}{2 \alpha^{\prime 2}} \int_{1}^{\infty} F\left(y_{4}\right) \mathrm{d} y_{4}, \\
& g_{\mathrm{op}}^{4} C_{D_{2}} \overline{\mathcal{A}}_{\mathrm{op}}[1423]=\frac{g_{\mathrm{op}}^{2}}{2 \alpha^{\prime 2}} \int_{0}^{1} F\left(y_{4}\right) \mathrm{d} y_{4}, \\
& g_{\mathrm{op}}^{4} C_{D_{2}} \overline{\mathcal{A}}_{\mathrm{op}}[4123]=\frac{g_{\mathrm{op}}^{2}}{2 \alpha^{\prime 2}} \int_{-\infty}^{0} F\left(y_{4}\right) \mathrm{d} y_{4},
\end{aligned}
$$

where the superscript "(4)" in each amplitude $\overline{\mathcal{A}}_{\text {op }}$ is not displayed for simplicity and the relation $g_{\mathrm{op}}^{2} C_{D_{2}}=1 /\left(2 \alpha^{\prime 2}\right)$ is imposed. In the above, the function $F\left(y_{4}\right)$ is defined as:

$$
\begin{aligned}
& F\left(y_{4}\right)=f\left(y_{4}\right)\left|y_{4}\right|^{-2 \alpha^{\prime}\left(\hat{k}_{1} \cdot \hat{k}_{4}\right)}\left|1-y_{4}\right|^{-2 \alpha^{\prime}\left(\hat{k}_{1} \cdot \hat{k}_{3}\right)}, \\
& f\left(y_{4}\right)=\lim _{y_{1} \rightarrow 0} \lim _{y_{2} \rightarrow 1} \lim _{y_{3} \rightarrow \infty} y_{3}^{2} \prod_{i \neq j} \exp \left[\frac{2 \alpha^{\prime}\left(\zeta_{i} \cdot \zeta_{j}\right)}{\left(y_{i}-y_{j}\right)^{2}}-\frac{2 \alpha^{\prime}\left(\zeta_{i} \cdot \hat{k}_{j}\right)}{y_{i}-y_{j}}\right],
\end{aligned}
$$




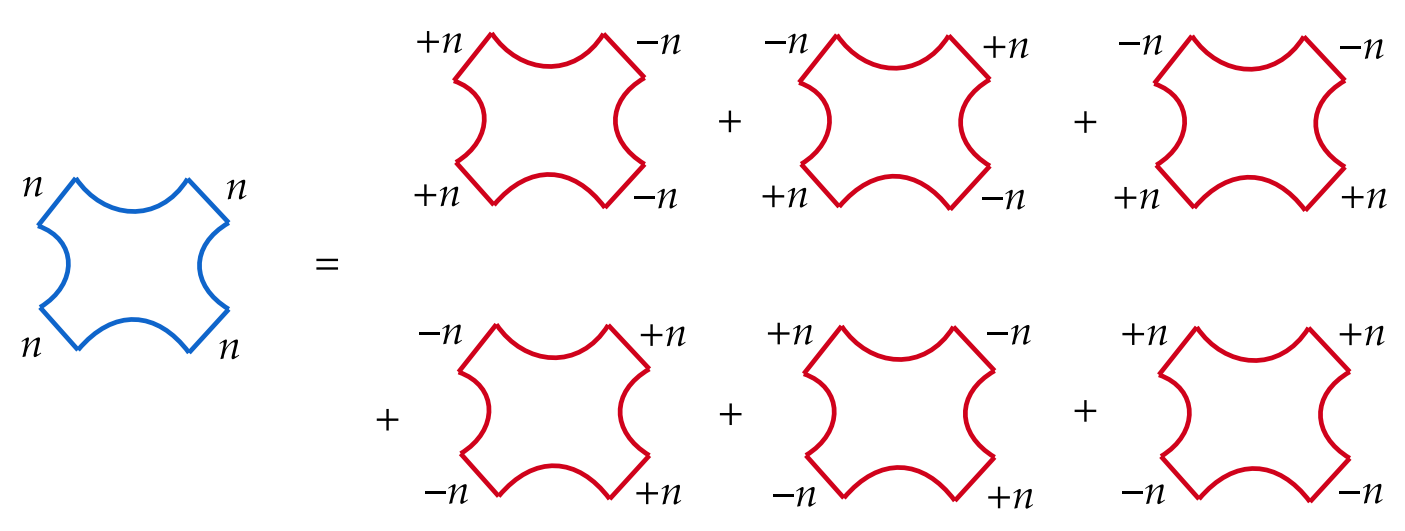

Figure 1. Elastic scattering amplitude of $(n, n) \rightarrow(n, n)$ for massive KK open strings with $\mathbb{Z}_{2}$-even parity (blue color) can be decomposed to a sum of six sub-amplitudes of massive KK open strings (red color) under the $\mathbb{S}^{1}$ compactification of $26 \mathrm{~d}$ for bosonic strings.

where we only need to expand up to the linear term of each external polarization vector. The other three color-ordered partial amplitudes ([1342], [1432], [4132]) can be obtained by exchanging $(2,3)$. In this section, we focus on the two partial amplitudes with the color-ordering [1234] and [1243] for the sake of our later double-copy construction closedstring amplitudes.

\subsection{Elastic amplitudes of KK gauge bosons from KK open strings}

In this subsection, we study the four-point elastic KK scattering process $(n, n) \rightarrow(n, n)$ with all external states being $\mathbb{Z}_{2}$-even. We observe that eq. (2.18) allows six different combinations of the KK numbers for the external states of the four-point sub-amplitudes (which are originally defined under the $\mathbb{S}^{1}$ compactification):

$$
\begin{aligned}
& \{+n,+n,-n,-n\},\{+n,-n,+n,-n\},\{+n,-n,-n,+n\}, \\
& \{-n,-n,+n,+n\},\{-n,+n,-n,+n\},\{-n,+n,+n,-n\},
\end{aligned}
$$

with $n \in \mathbb{Z}^{+}$. This means that the four-point elastic KK open-string amplitude $\mathcal{A}_{\mathrm{op}}^{(4)}$ can be decomposed into a sum of the six sub-amplitudes, as presented in figure 1 . We note that the compactification under $\mathbb{S}^{1}$ respects the $\mathbb{Z}_{2}$ parity, so among the above six combinations of KK numbers only three are independent, where the three combinations in the first row of eq. (3.3) are connected to the other three corresponding combinations in the second row by $\mathbb{Z}_{2}$ parity transformation. From the above, our key insight is that even though the external states on the l.h.s. of eq.(3.3) are all $\mathbb{Z}_{2}$-even, the external states on the r.h.s. contain two $\mathbb{Z}_{2}$-even states and two $\mathbb{Z}_{2}$-odd states such that the condition (2.18) is obeyed. This is because our string compactification of $26 \mathrm{~d}$ is under $\mathbb{S}^{1}$ with the periodic boundary condition (2.1) (without having $\mathbb{Z}_{2}$ orbifold). Hence, even for a scattering amplitude with $\mathbb{Z}_{2}$-even external states $(2.17 \mathrm{a})$, it contains the combination of individual amplitudes whose external states include both positive and negative KK numbers, as shown in eq. (3.3) for the case of four-point amplitudes. 
Then, we compute the color-ordered partial amplitudes from eq. (3.1) under the field theory limit $\alpha^{\prime} \rightarrow 0$. We present their explicit expressions in appendix $\mathrm{B}$, where the openstring coupling $g_{\text {op }}$ is replaced by the gauge coupling $g$ of the YM theory. Thus, substituting the momenta in eq. (A.1) and the longitudinal polarization vectors $\zeta_{j, L}^{\mu}$ in eq. (A.5) into eq. (3.26), we derive the sub-amplitudes with color ordering [1234]:

$$
\begin{aligned}
& \mathcal{T}\left[1_{L}^{ \pm n} 2_{L}^{ \pm n} 3_{L}^{\mp n} 4_{L}^{\mp n}\right]=g^{2} \frac{7+c_{2 \theta}}{\left(1+c_{\theta}\right)}, \\
& \mathcal{T}\left[1_{L}^{ \pm n} 2_{L}^{\mp n} 3_{L}^{ \pm n} 4_{L}^{\mp n}\right]=g^{2} \frac{\left[7 \bar{s}^{2}-24 \bar{s}+48-16(\bar{s}-4) c_{\theta}+(\bar{s}+4)^{2} c_{2 \theta}\right] \sec ^{2} \frac{\theta}{2}}{2 \bar{s}(\bar{s}-4)}, \\
& \mathcal{T}\left[1_{L}^{ \pm n} 2_{L}^{\mp n} 3_{L}^{\mp n} 4_{L}^{ \pm n}\right]=g^{2} \frac{7 \bar{s}^{2}-24 \bar{s}+48+16(\bar{s}-4) c_{\theta}+(\bar{s}+4)^{2} c_{2 \theta}}{\bar{s}\left[\bar{s}+4+(\bar{s}-4) c_{\theta}\right]},
\end{aligned}
$$

and the sub-amplitudes with color ordering [1243]:

$$
\begin{aligned}
& \mathcal{T}\left[1_{L}^{ \pm n} 2_{L}^{ \pm n} 4_{L}^{\mp n} 3_{L}^{\mp n}\right]=g^{2} \frac{\left(7+c_{2 \theta}\right) \csc ^{2} \frac{\theta}{2}}{2} \\
& \mathcal{T}\left[1_{L}^{ \pm n} 2_{L}^{\mp n} 4_{L}^{ \pm n} 3_{L}^{\mp n}\right]=g^{2} \frac{7 \bar{s}^{2}-24 \bar{s}+48-16(\bar{s}-4) c_{\theta}+(\bar{s}+4)^{2} c_{2 \theta}}{\bar{s}\left[\bar{s}+4-(\bar{s}-4) c_{\theta}\right]} \\
& \mathcal{T}\left[1_{L}^{ \pm n} 2_{L}^{\mp n} 4_{L}^{\mp n} 3_{L}^{ \pm n}\right]=g^{2} \frac{\left[7 \bar{s}^{2}-24 \bar{s}+48+16(\bar{s}-4) c_{\theta}+(\bar{s}+4)^{2} c_{2 \theta}\right] \csc ^{2} \frac{\theta}{2}}{2 \bar{s}(\bar{s}-4)}
\end{aligned}
$$

where we have defined $\bar{s}=s / M_{n}^{2}$. With the above, we sum up the four-point amplitudes in eq. (3.4) and eq. (3.5), and derive the following color-ordered full elastic amplitudes with all external states being $\mathbb{Z}_{2}$ even:

$$
\begin{aligned}
\mathcal{T}\left[1_{L}^{n} 2_{L}^{n} 3_{L}^{n} 4_{L}^{n}\right] & =\frac{1}{2}\left(\mathcal{T}\left[1_{L}^{+n} 2_{L}^{+n} 3_{L}^{-n} 4_{L}^{-n}\right]+\mathcal{T}\left[1_{L}^{+n} 2_{L}^{-n} 3_{L}^{+n} 4_{L}^{-n}\right]+\mathcal{T}\left[1_{L}^{+n} 2_{L}^{-n} 3_{L}^{-n} 4_{L}^{+n}\right]\right) \\
& =g^{2} \frac{\left(P_{0}+P_{1} c_{\theta}+P_{2} c_{2 \theta}+P_{3} c_{3 \theta}\right) \sec ^{2} \frac{\theta}{2}}{16 \bar{s}(\bar{s}-4)\left[\bar{s}+4+(\bar{s}-4) c_{\theta}\right]} \\
\mathcal{T}\left[1_{L}^{n} 2_{L}^{n} 4_{L}^{n} 3_{L}^{n}\right] & =\frac{1}{2}\left(\mathcal{T}\left[1_{L}^{+n} 2_{L}^{+n} 4_{L}^{-n} 3_{L}^{-n}\right]+\mathcal{T}\left[1_{L}^{+n} 2_{L}^{-n} 4_{L}^{+n} 3_{L}^{-n}\right]+\mathcal{T}\left[1_{L}^{+n} 2_{L}^{-n} 4_{L}^{-n} 3_{L}^{+n}\right]\right) \\
& =g^{2} \frac{\left(P_{0}-P_{1} c_{\theta}+P_{2} c_{2 \theta}-P_{3} c_{3 \theta}\right) \csc ^{2} \frac{\theta}{2}}{16 \bar{s}(\bar{s}-4)\left[\bar{s}+4-(\bar{s}-4) c_{\theta}\right]}
\end{aligned}
$$

where the polynomials $\left\{P_{j}\right\}$ are given by

$$
\begin{array}{ll}
P_{0}=42 \bar{s}^{3}-96 \bar{s}^{2}-32 \bar{s}, & P_{1}=45 \bar{s}^{3}-320 \bar{s}^{2}+528 \bar{s}+128, \\
P_{2}=2 \bar{s}\left(3 \bar{s}^{2}+16 \bar{s}+16\right), & P_{3}=3 \bar{s}^{3}-16 \bar{s}-128 .
\end{array}
$$

The above elastic KK gauge boson amplitudes (3.6a)-(3.6b) are derived from the KK openstring amplitudes (3.1). We inspect these color-ordered KK gauge boson amplitudes (3.6) based on the KK open-string calculation and find that they can be expressed in the following forms:

$$
\mathcal{T}\left[1_{L}^{n} 2_{L}^{n} 3_{L}^{n} 4_{L}^{n}\right]=g^{2}\left(-\mathcal{K}_{s}^{\mathrm{el}}+\mathcal{K}_{t}^{\mathrm{el}}\right), \quad \mathcal{T}\left[1_{L}^{n} 2_{L}^{n} 4_{L}^{n} 3_{L}^{n}\right]=g^{2}\left(\mathcal{K}_{s}^{\mathrm{el}}-\mathcal{K}_{u}^{\mathrm{el}}\right),
$$




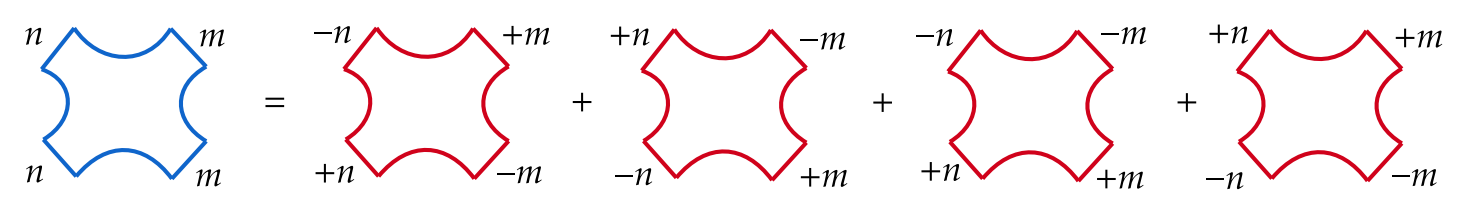

Figure 2. Inelastic scattering amplitude of $(n, n) \rightarrow(m, m)$ for massive KK open strings with $\mathbb{Z}_{2}$-even parity (blue color) can be decomposed to a sum of four sub-amplitudes of massive KK open strings (red color) under the $\mathbb{S}^{1}$ compactification of $26 \mathrm{~d}$ for bosonic strings.

where the kinematic factors $\left\{\mathcal{K}_{j}^{\mathrm{el}}\right\}$ are summarized in eqs. (B.2)-(B.3) of appendix B. Impressively, we find that the expressions in eq. (3.8) fully agree with the corresponding KK gauge boson amplitudes of ref. [32] which were computed independently within the compactified 5d KK YM gauge field theory.

\subsection{Inelastic amplitudes of KK gauge bosons from KK open strings}

Next, we analyze the four-point color-ordered partial amplitude for the inelastic channel $(n, n) \rightarrow(m, m)$ with all external states being $\mathbb{Z}_{2}$-even. We inspect eq. (2.18) and find that different from the elastic channel, there are only four allowed combinations of the external KK states (as originally defined under the $\mathbb{S}^{1}$ compactification):

$$
\begin{aligned}
& \{+n,-n,+m,-m\},\{+n,-n,-m,+m\}, \\
& \{-n,+n,-m,+m\},\{-n,+n,+m,-m\} .
\end{aligned}
$$

Thus, the four-point inelastic KK open-string amplitude is given by a sum of the four sub-amplitudes, as illustrated in figure 2.

Then, we derive the four-point inelastic scattering amplitudes of longitudinal gauge bosons with color-ordering [1234]:

$$
\begin{aligned}
\mathcal{T}\left[1_{L}^{ \pm n} 2_{L}^{\mp n} 3_{L}^{ \pm m} 4_{L}^{\mp m}\right] & =g^{2} \frac{\left(7 \bar{s}^{2}-12 r_{+}^{2} \bar{s}+48 r^{2}\right)-64 r \bar{q} \bar{q}^{\prime} c_{\theta}+16 \bar{q}^{2} \bar{q}^{\prime 2} c_{2 \theta}}{2 \bar{s}\left[(\bar{s}-4 r)+4 \bar{q} \bar{q}^{\prime} c_{\theta}\right]}, \\
\mathcal{T}\left[1_{L}^{ \pm n} 2_{L}^{\mp n} 3_{L}^{\mp m} 4_{L}^{ \pm m}\right] & =g^{2} \frac{\left(7 \bar{s}^{2}-12 r_{+}^{2} \bar{s}+48 r^{2}\right)+64 r \bar{q} \bar{q}^{\prime} c_{\theta}+16 \bar{q}^{2} \bar{q}^{\prime 2} c_{2 \theta}}{2 \bar{s}\left[(\bar{s}+4 r)+4 \bar{q} \bar{q}^{\prime} c_{\theta}\right]},
\end{aligned}
$$

where we have used the notations,

$$
\begin{aligned}
& q=\left(E^{2}-M_{n}^{2}\right)^{\frac{1}{2}}, \quad q^{\prime}=\left(E^{2}-M_{m}^{2}\right)^{\frac{1}{2}}, \quad r=M_{m} / M_{n}, \quad r_{+}^{2}=1+r^{2}, \\
& s=4 E^{2}, \quad s_{0}=4 q^{2}, \quad \bar{s}=s / M_{n}^{2}=\bar{s}_{0}+4, \quad \bar{s}_{0}=s_{0} / M_{n}^{2}, \\
& \bar{q}^{2}=q^{2} / M_{n}^{2}=\bar{s} / 4-1, \quad \bar{q}^{2}=q^{\prime 2} / M_{n}^{2}=\bar{s} / 4-r^{2}, \\
& \bar{q}^{2} \bar{q}^{2}=(\bar{s}-4)\left(\bar{s}-4 r^{2}\right) / 16 .
\end{aligned}
$$

For the color-ordering [1243], we derive the four-point inelastic scattering amplitudes of longitudinal gauge bosons as follows:

$$
\begin{aligned}
\mathcal{T}\left[1_{L}^{ \pm n} 2_{L}^{\mp n} 4_{L}^{ \pm m} 3_{L}^{\mp m}\right] & =g^{2} \frac{\left(7 \bar{s}^{2}-12 r_{+}^{2} \bar{s}+48 r^{2}\right)-64 r \bar{q} \bar{q}^{\prime} c_{\theta}+16 \bar{q}^{2} \bar{q}^{\prime 2} c_{2 \theta}}{2 \bar{s}\left[(\bar{s}+4 r)-4 \bar{q} \bar{q}^{\prime} c_{\theta}\right]} \\
\mathcal{T}\left[1_{L}^{ \pm n} 2_{L}^{\mp n} 4_{L}^{\mp m} 3_{L}^{ \pm m}\right] & =g^{2} \frac{\left(7 \bar{s}^{2}-12 r_{+}^{2} \bar{s}+48 r^{2}\right)+64 r \bar{q} \bar{q}^{\prime} c_{\theta}+16 \bar{q}^{2} \bar{q}^{\prime 2} c_{2 \theta}}{2 \bar{s}\left[(\bar{s}-4 r)-4 \bar{q} \bar{q}^{\prime} c_{\theta}\right]}
\end{aligned}
$$




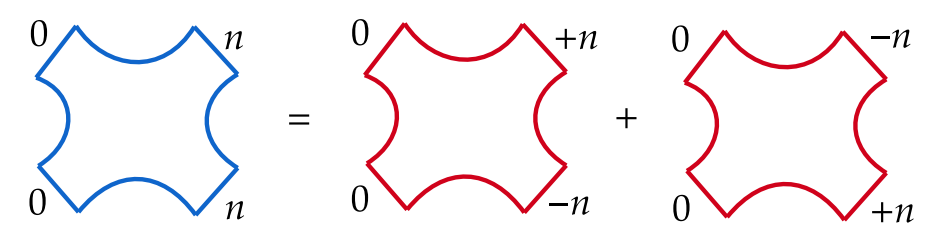

Figure 3. Inelastic scattering amplitudes of $(0,0) \rightarrow(n, n)$ for massive KK open string with $\mathbb{Z}_{2^{-}}$ even parity (blue color) can be decomposed into a sum of two sub-amplitudes of massive KK open strings (red color) under the $\mathbb{S}^{1}$ compactification of $26 \mathrm{~d}$ for bosonic strings.

Summing up the four-point inelastic amplitudes (3.10) and (3.12), we derive the following color-ordered full amplitudes with all external states being $\mathbb{Z}_{2}$ even:

$$
\begin{aligned}
\mathcal{T}\left[1_{L}^{n} 2_{L}^{n} 3_{L}^{m} 4_{L}^{m}\right] & =\frac{1}{2}\left(\mathcal{T}\left[1_{L}^{+n} 2_{L}^{-n} 3_{L}^{+m} 4_{L}^{-m}\right]+\mathcal{T}\left[1_{L}^{+n} 2_{L}^{-n} 3_{L}^{-m} 4_{L}^{+m}\right]\right) \\
& =g^{2} \frac{P_{0}+P_{1} c_{\theta}+P_{2} c_{2 \theta}+P_{3} c_{3 \theta}}{\bar{s}\left(3 \bar{s}^{2}-4 \bar{s} r_{+}^{2}-16 r^{2}+16 \bar{s} \bar{q} \bar{q}^{\prime} c_{\theta}+16 \bar{q}^{2} \bar{q}^{\prime 2} c_{2 \theta}\right)}, \\
\mathcal{T}\left[1_{L}^{n} 2_{L}^{n} 4_{L}^{m} 3_{L}^{m}\right] & =\frac{1}{2}\left(\mathcal{T}\left[1_{L}^{+n} 2_{L}^{-n} 4_{L}^{+m} 3_{L}^{-m}\right]+\mathcal{T}\left[1_{L}^{+n} 2_{L}^{-n} 4_{L}^{-m} 3_{L}^{+m}\right]\right) \\
& =g^{2} \frac{P_{0}-P_{1} c_{\theta}+P_{2} c_{2 \theta}-P_{3} c_{3 \theta}}{\bar{s}\left(3 \bar{s}^{2}-4 \bar{s} r_{+}^{2}-16 r^{2}-16 \bar{s} \bar{q} \bar{q}^{\prime} c_{\theta}+16 \bar{q}^{2} \bar{q}^{\prime 2} c_{2 \theta}\right)},
\end{aligned}
$$

where the polynomials $\left\{P_{j}\right\}$ take the forms:

$$
\begin{array}{ll}
P_{0}=\bar{s}\left(7 \bar{s}^{2}-12 \bar{s} r_{+}^{2}+48 r^{2}\right), & P_{1}=2 \bar{q} \bar{q}^{\prime}\left(15 \bar{s}^{2}-20 \bar{s} r_{+}^{2}-16 r^{2}\right), \\
P_{2}=\bar{s}\left(\bar{s}^{2}+4 \bar{s} r_{+}^{2}+16 r^{2}\right), & P_{3}=2 \bar{q} \bar{q}^{\prime}\left(\bar{s}^{2}+4 \bar{s} r_{+}^{2}+16 r^{2}\right) .
\end{array}
$$

Inspecting the string-based KK gauge boson amplitudes (3.13), we can re-express them in the following forms:

$$
\mathcal{T}\left[1_{L}^{n} 2_{L}^{n} 3_{L}^{m} 4_{L}^{m}\right]=g^{2}\left(-\mathcal{K}_{s}^{\mathrm{in}}+\mathcal{K}_{t}^{\mathrm{in}}\right), \quad \mathcal{T}\left[1_{L}^{n} 2_{L}^{n} 4_{L}^{m} 3_{L}^{m}\right]=g^{2}\left(\mathcal{K}_{s}^{\mathrm{in}}-\mathcal{K}_{u}^{\mathrm{in}}\right),
$$

where the kinematic functions $\left\{\mathcal{K}_{j}^{\text {in }}\right\}$ are summarized in eqs. (B.15)-(B.16) of appendix B. We find that these $\left\{\mathcal{K}_{j}^{\text {in }}\right\}$ functions fully agree with what we derived independently from the compactified 5d KK YM gauge field theory.

Next, we study the mixed inelastic channel of gauge boson scattering $(0,0) \rightarrow(n, n)$. We find that the condition (2.18) allows only two combinations of the external KK states (as originally defined under the $\mathbb{S}^{1}$ compactification of $26 \mathrm{~d}$ bosonic strings),

$$
\left\{n_{1}, n_{2}, n_{3}, n_{4}\right\}=\{0,0,+n,-n\},\{0,0,-n,+n\} .
$$

Hence, this inelastic KK open-string amplitude equals a sum of six sub-amplitudes, as shown in figure 3. Then, we compute the color-ordered gauge boson amplitudes as follows:

$$
\begin{aligned}
& \mathcal{T}\left[1_{+1}^{0} 2_{-1}^{0} 3_{L}^{ \pm n} 4_{L}^{\mp n}\right]=-\frac{g^{2}(\bar{s}+4) s_{\theta}^{2}}{\bar{s}+2 \bar{s}^{1 / 2} \bar{q} c_{\theta}}, \\
& \mathcal{T}\left[1_{+1}^{0} 2_{-1}^{0} 4_{L}^{ \pm n} 3_{L}^{\mp n}\right]=-\frac{g^{2}(\bar{s}+4) s_{\theta}^{2}}{\bar{s}-2 \bar{s}^{1 / 2} \bar{q} c_{\theta}},
\end{aligned}
$$

where $\bar{s}$ and $\bar{q}$ are defined in eq. (3.11). 
Then, from the four-point inelastic amplitudes in eq. (3.17), we can obtain the following color-ordered amplitudes with all external states being $\mathbb{Z}_{2}$ even:

$$
\begin{gathered}
\mathcal{T}\left[1_{+1}^{0} 2_{-1}^{0} 3_{L}^{n} 4_{L}^{n}\right]=\mathcal{T}\left[1_{+1}^{0} 2_{-1}^{0} 3_{L}^{+n} 4_{L}^{-n}\right]=-\frac{g^{2}(\bar{s}+4) s_{\theta}^{2}}{\bar{s}+2 \bar{s}^{1 / 2} \bar{q} c_{\theta}} \\
\mathcal{T}\left[1_{+1}^{0} 2_{-1}^{0} 4_{L}^{n} 3_{L}^{n}\right]=\mathcal{T}\left[1_{+1}^{0} 2_{-1}^{0} 4_{L}^{+n} 3_{L}^{-n}\right]=-\frac{g^{2}(\bar{s}+4) s_{\theta}^{2}}{\bar{s}-2 \bar{s}^{1 / 2} \bar{q} c_{\theta}}
\end{gathered}
$$

We can re-express the above string-based inelastic amplitudes in the following forms:

$$
\mathcal{T}\left[1_{+1}^{0} 2_{-1}^{0} 3_{L}^{n} 4_{L}^{n}\right]=g^{2}\left(-\mathcal{K}_{s}^{\text {in }}+\mathcal{K}_{t}^{\text {in }}\right), \quad \mathcal{T}\left[1_{+1}^{0} 2_{-1}^{0} 4_{L}^{n} 3_{L}^{n}\right]=g^{2}\left(\mathcal{K}_{s}^{\text {in }}-\mathcal{K}_{u}^{\text {in }}\right),
$$

where the kinematic functions $\left\{\mathcal{K}_{j}^{\text {in }}\right\}$ are summarized in eq. (B.29) of appendix B and fully agree with what we derived independently from the compactified 5d KK YM gauge field theory.

\subsection{Structure of color-ordered massive KK amplitudes}

In this subsection, we study the structure of the color-ordered scattering amplitudes of massive KK gauge bosons. We demonstrate that the tree-level massive KK gauge boson amplitudes can be obtained from the corresponding color-ordered amplitudes of the massless zero-mode gauge bosons by making proper shifts of the Mandelstam variables.

From the formulation of the open-string amplitudes in section 2, we observe that colorordered massive KK sub-amplitudes in $d$-dimensions, such as $\mathcal{T}\left[1^{+n} 2^{+n} 3^{-n} 4^{-n}\right]$, can be viewed as the massless amplitudes in $(d+1)$-dimensions with the $(d+1)$-th component of each momentum being discretized since the $(d+1)$-th spatial dimension is compactified on $\mathbb{S}^{1}$. Namely, we can express the $(d+1)$-dimensional momentum $\hat{k}^{\hat{\mu}}$ in terms of the $d$ dimensional momentum $k^{\mu}$ plus an extra discretized $(d+1)$-th component: $\hat{k}^{\hat{\mu}}=\left(k^{\mu}, \hat{n} / R\right)$. For a given polarization vector $\zeta^{\mu}$ of the on-shell gauge boson in $d$-dimensions, we can symbolically express it as a $(d+1)$-dimensional polarization vector $\hat{\zeta}^{\hat{\mu}}=\left(\zeta^{\mu}, 0\right)$. Thus, we have $\hat{\zeta}_{i} \cdot \hat{\zeta}_{j}=\zeta_{i} \cdot \zeta_{j}$ and $\hat{\zeta}_{i} \cdot \hat{k}_{j}=\zeta_{i} \cdot k_{j}$, where the subscripts $(i, j)$ denote the particle numbers of the external states. ${ }^{2}$ Keeping these in mind, we can first compute a $(d+1)$-dimensional massless scattering amplitude and then we deduce the corresponding $d$-dimensional massive KK amplitude by using relations

$$
\hat{\zeta}_{i} \cdot \hat{\zeta}_{j}=\zeta_{i} \cdot \zeta_{j}, \quad \hat{\zeta}_{i} \cdot \hat{k}_{j}=\zeta_{i} \cdot k_{j}
$$

and the relation between the two sets of Mandelstam variables

$$
\hat{s}_{i j}=-2 \hat{k}_{i} \cdot \hat{k}_{j}=s_{i j}-\left(\frac{\hat{n}_{i}+\hat{n}_{j}}{R}\right)^{2}
$$

\footnotetext{
${ }^{2}$ Our later explicit calculations of the KK scattering amplitudes will be always performed in the effective $(3+1)$-dimensional spacetime with $d=4$, and with a single compactified extra spatial dimension of coordinate $X^{25}$. The other extra spatial dimensions of coordinates $\left\{X^{4}, \cdots, X^{24}\right\}$ have much smaller radii $r_{j}=\mathcal{O}\left(M_{\mathrm{Pl}}^{-1}\right)(j=4, \cdots, 24)$, so they are fully decoupled at energy scales much below the reduced Planck scale $M_{\mathrm{Pl}}$, as we discussed at the beginning of section 2.1. Thus, the bosonic strings effectively propagate in $(4+1) d$ spacetime with the single extra spatial dimension of $X^{25}$ compactified on $\mathbb{S}^{1}$.
} 
where the $(d+1)$-dimensional momenta obey the on-shell conditions $\hat{k}_{i}^{2}=\hat{k}_{j}^{2}=0$. Optionally, we can first write a $d$-dimensional massless (zero-mode) scattering amplitude $\mathcal{T}_{(0)}\left(s_{i j}\right)$ with all polarization vectors and momenta of the external states in symbolic format, and then we deduce the corresponding KK sub-amplitude $\mathcal{T}_{\mathrm{KK}}^{\mathrm{sub}}\left(s_{i j}\right)$ as follows:

$$
\mathcal{T}_{\mathrm{KK}}^{\mathrm{sub}}\left(s_{i j}\right)=\mathcal{T}_{(0)}\left(\hat{s}_{i j}\right),
$$

with each $d$-dimensional external state having its momentum obey the on-shell condition of the massive KK gauge boson $\left(k_{j}^{2}=-M_{j}^{2}\right)$ and its polarization vector replaced by the polarization vector $\zeta_{j}$ of the KK gauge boson. In this way, we can derive all the $d$-dimensional massive KK gauge boson amplitudes according to the structure of the corresponding $d$ dimensional massless (zero-mode) gauge boson amplitudes.

Next, we compute a four-point massless (zero-mode) gauge boson scattering amplitude with color ordering [1234]. This can be done either in the massless YM field theory, or, we can deduce it by taking the field theory limit $\alpha^{\prime} \rightarrow 0$ of the open-string scattering amplitude (3.1):

$$
\begin{aligned}
\mathcal{T}\left[1^{0} 2^{0} 3^{0} 4^{0}\right] & \\
= & g^{2}\left\{2\left[\left(\zeta_{1} \cdot \zeta_{3}\right)\left(\zeta_{2} \cdot \zeta_{4}\right)-\left(\zeta_{1} \cdot \zeta_{4}\right)\left(\zeta_{2} \cdot \zeta_{3}\right)-\left(\zeta_{1} \cdot \zeta_{2}\right)\left(\zeta_{3} \cdot \zeta_{4}\right)\right]\right. \\
& +\frac{4}{t}\left[\left(\zeta_{2} \cdot \zeta_{3}\right)\left(\zeta_{1} \cdot k_{2}\right)\left(\zeta_{4} \cdot k_{1}\right)-\left(\zeta_{2} \cdot \zeta_{3}\right)\left(\zeta_{1} \cdot k_{4}\right)\left(\zeta_{4} \cdot k_{2}\right)-\left(\zeta_{1} \cdot \zeta_{4}\right)\left(\zeta_{2} \cdot k_{4}\right)\left(\zeta_{3} \cdot k_{2}\right)\right. \\
& +\left(\zeta_{2} \cdot \zeta_{4}\right)\left(\zeta_{1} \cdot k_{4}\right)\left(\zeta_{3} \cdot k_{2}\right)-\left(\zeta_{1} \cdot \zeta_{4}\right)\left(\zeta_{2} \cdot k_{1}\right)\left(\zeta_{3} \cdot k_{4}\right)-\left(\zeta_{1} \cdot \zeta_{4}\right)\left(\zeta_{2} \cdot k_{4}\right)\left(\zeta_{3} \cdot k_{4}\right) \\
& +\left(\zeta_{3} \cdot \zeta_{4}\right)\left(\zeta_{1} \cdot k_{4}\right)\left(\zeta_{2} \cdot k_{1}\right)+\left(\zeta_{3} \cdot \zeta_{4}\right)\left(\zeta_{1} \cdot k_{4}\right)\left(\zeta_{2} \cdot k_{4}\right)-\left(\zeta_{1} \cdot \zeta_{3}\right)\left(\zeta_{2} \cdot k_{1}\right)\left(\zeta_{4} \cdot k_{1}\right) \\
& \left.-\left(\zeta_{1} \cdot \zeta_{3}\right)\left(\zeta_{2} \cdot k_{4}\right)\left(\zeta_{4} \cdot k_{1}\right)-\left(\zeta_{1} \cdot \zeta_{2}\right)\left(\zeta_{3} \cdot k_{2}\right)\left(\zeta_{4} \cdot k_{1}\right)-\frac{s}{2}\left(\zeta_{1} \cdot \zeta_{4}\right)\left(\zeta_{2} \cdot \zeta_{3}\right)\right] \\
& +\frac{4}{s}\left[\left(\zeta_{2} \cdot \zeta_{4}\right)\left(\zeta_{1} \cdot k_{2}\right)\left(\zeta_{3} \cdot k_{4}\right)-\left(\zeta_{1} \cdot \zeta_{4}\right)\left(\zeta_{2} \cdot k_{1}\right)\left(\zeta_{3} \cdot k_{4}\right)-\left(\zeta_{1} \cdot \zeta_{2}\right)\left(\zeta_{4} \cdot k_{2}\right)\left(\zeta_{3} \cdot k_{4}\right)\right. \\
& +\left(\zeta_{3} \cdot \zeta_{4}\right)\left(\zeta_{1} \cdot k_{4}\right)\left(\zeta_{2} \cdot k_{1}\right)-\left(\zeta_{3} \cdot \zeta_{4}\right)\left(\zeta_{1} \cdot k_{2}\right)\left(\zeta_{2} \cdot k_{4}\right)-\left(\zeta_{1} \cdot \zeta_{3}\right)\left(\zeta_{2} \cdot k_{1}\right)\left(\zeta_{4} \cdot k_{1}\right) \\
& +\left(\zeta_{2} \cdot \zeta_{3}\right)\left(\zeta_{1} \cdot k_{2}\right)\left(\zeta_{4} \cdot k_{1}\right)-\left(\zeta_{1} \cdot \zeta_{2}\right)\left(\zeta_{3} \cdot k_{2}\right)\left(\zeta_{4} \cdot k_{1}\right)-\left(\zeta_{1} \cdot \zeta_{3}\right)\left(\zeta_{2} \cdot k_{1}\right)\left(\zeta_{4} \cdot k_{2}\right) \\
& \left.\left.+\left(\zeta_{2} \cdot \zeta_{3}\right)\left(\zeta_{1} \cdot k_{2}\right)\left(\zeta_{4} \cdot k_{2}\right)-\left(\zeta_{1} \cdot \zeta_{2}\right)\left(\zeta_{3} \cdot k_{2}\right)\left(\zeta_{4} \cdot k_{2}\right)-\frac{t}{2}\left(\zeta_{1} \cdot \zeta_{2}\right)\left(\zeta_{3} \cdot \zeta_{4}\right)\right]\right\}
\end{aligned}
$$

For the other massless color-ordered amplitudes, such as the one with color ordering [1243], they can be obtained through the relation:

$$
u \mathcal{T}\left[1^{0} 2^{0} 4^{0} 3^{0}\right]=t \mathcal{T}\left[1^{0} 2^{0} 3^{0} 4^{0}\right]
$$

For instance, we consider the elastic KK scattering $(n, n) \rightarrow(n, n)$ as discussed in section 3.1. We can obtain the color-ordered sub-amplitude $\mathcal{T}\left[1^{+n} 2^{+n} 3^{-n} 4^{-n}\right]$ by the replacement $s \rightarrow\left(s-4 M_{n}^{2}\right)$ in $\mathcal{T}\left[1^{0} 2^{0} 3^{0} 4^{0}\right]$. In general, the color-ordered sub-amplitude 
$\mathcal{T}\left[1^{\hat{n}_{1}} 2^{\hat{n}_{2}} 3^{\hat{n}_{3}} 4^{\hat{n}_{4}}\right]$ can be obtained by the following replacements:

$$
s_{i j} \longrightarrow s_{i j}-\left(\frac{\hat{n}_{i}+\hat{n}_{j}}{R}\right)^{2} .
$$

This procedure can be applied to deriving the general $N$-point scattering amplitudes of massive KK gauge bosons and be extended to the case of KK gravitons which we will present elsewhere.

For the color-ordered combinations [1234] and [1243], we derive the following massive KK gauge boson amplitudes according to eq. (3.3):

$$
\begin{aligned}
\mathcal{T}\left[1^{n} 2^{n} 3^{n} 4^{n}\right] & =\frac{1}{2}\left(\mathcal{T}_{1}^{\mathrm{el}}+\mathcal{T}_{2}^{\mathrm{el}}+\mathcal{T}_{3}^{\mathrm{el}}\right) \\
\mathcal{T}\left[1^{n} 2^{n} 4^{n} 3^{n}\right] & =\frac{1}{2}\left(\frac{t}{u} \mathcal{T}_{1}^{\mathrm{el}}+\frac{t}{u-4 M_{n}^{2}} \mathcal{T}_{2}^{\mathrm{el}}+\frac{t-4 M_{n}^{2}}{u} \mathcal{T}_{3}^{\mathrm{el}}\right)
\end{aligned}
$$

where the basis amplitudes $\left\{\mathcal{T}_{1}^{\mathrm{el}}, \mathcal{T}_{2}^{\mathrm{el}}, \mathcal{T}_{3}^{\mathrm{el}}\right\}$ are given by the following sub-amplitudes:

$$
\begin{aligned}
& \mathcal{T}_{1}^{\mathrm{el}}=\mathcal{T}\left[1^{ \pm n} 2^{ \pm n} 3^{\mp n} 4^{\mp n}\right]=\left.\mathcal{T}\left[1^{0} 2^{0} 3^{0} 4^{0}\right]\right|_{s \rightarrow\left(s-4 M_{n}^{2}\right)}, \\
& \mathcal{T}_{2}^{\mathrm{el}}=\mathcal{T}\left[1^{ \pm n} 2^{\mp n} 3^{ \pm n} 4^{\mp n}\right]=\left.\mathcal{T}\left[1^{0} 2^{0} 3^{0} 4^{0}\right]\right|_{u \rightarrow\left(u-4 M_{n}^{2}\right)}, \\
& \mathcal{T}_{3}^{\mathrm{el}}=\mathcal{T}\left[1^{ \pm n} 2^{\mp n} 3^{\mp n} 4^{ \pm n}\right]=\left.\mathcal{T}\left[1^{0} 2^{0} 3^{0} 4^{0}\right]\right|_{t \rightarrow\left(t-4 M_{n}^{2}\right)}
\end{aligned}
$$

We note that the KK numbers of the sub-amplitude $\mathcal{T}\left[1^{ \pm n} 2^{\mp n} 3^{ \pm n} 4^{\mp n}\right]$ in eq. (3.27b) makes the shifted mass-term in eq. (3.25) vanish, so there is practically no replacement needed.

Following the same procedure, we derive the following color-ordered amplitudes for the inelastic scattering channel $(n, n) \rightarrow(m, m)$ :

$$
\begin{aligned}
\mathcal{T}\left[1^{n} 2^{n} 3^{m} 4^{m}\right] & =\frac{1}{2}\left(\mathcal{T}_{1}^{\mathrm{in}}+\mathcal{T}_{2}^{\mathrm{in}}\right), \\
\mathcal{T}\left[1^{n} 2^{n} 4^{m} 3^{m}\right] & =\frac{1}{2}\left(\frac{t-M_{n-m}^{2}}{u-M_{n+m}^{2}} \mathcal{T}_{1}^{\mathrm{in}}+\frac{t-M_{n+m}^{2}}{u-M_{n-m}^{2}} \mathcal{T}_{2}^{\mathrm{in}}\right),
\end{aligned}
$$

where the basis amplitudes $\left\{\mathcal{T}_{1}^{\text {in }}, \mathcal{T}_{2}^{\text {in }}\right\}$ are obtained by the relations:

$$
\begin{aligned}
& \mathcal{T}_{1}^{\text {in }}=\mathcal{T}\left[1^{ \pm n} 2^{\mp n} 3^{ \pm m} 4^{\mp m}\right]=\left.\mathcal{T}\left[1^{0} 2^{0} 3^{0} 4^{0}\right]\right|_{t \rightarrow\left(t-M_{n-m}^{2}\right)}, \\
& \mathcal{T}_{2}^{\text {in }}=\mathcal{T}\left[1^{ \pm n} 2^{\mp n} 3^{\mp m} 4^{ \pm m}\right]=\left.\mathcal{T}\left[1^{0} 2^{0} 3^{0} 4^{0}\right]\right|_{t \rightarrow\left(t-M_{n+m}^{2}\right)} .
\end{aligned}
$$

For the inelastic scattering channel $(0,0) \rightarrow(n, n)$, we can deduce its amplitude from that of $(n, n) \rightarrow(m, m)$ by the following replacements:

$$
\begin{aligned}
& \mathcal{T}\left[1^{0} 2^{0} 3^{n} 4^{n}\right]=\left.\mathcal{T}\left[1^{n} 2^{n} 3^{m} 4^{m}\right]\right|_{\{n \rightarrow 0, m \rightarrow n\}}, \\
& \mathcal{T}\left[1^{0} 2^{0} 4^{n} 3^{n}\right]=\left.\mathcal{T}\left[1^{n} 2^{n} 4^{m} 3^{m}\right]\right|_{\{n \rightarrow 0, m \rightarrow n\}}
\end{aligned}
$$



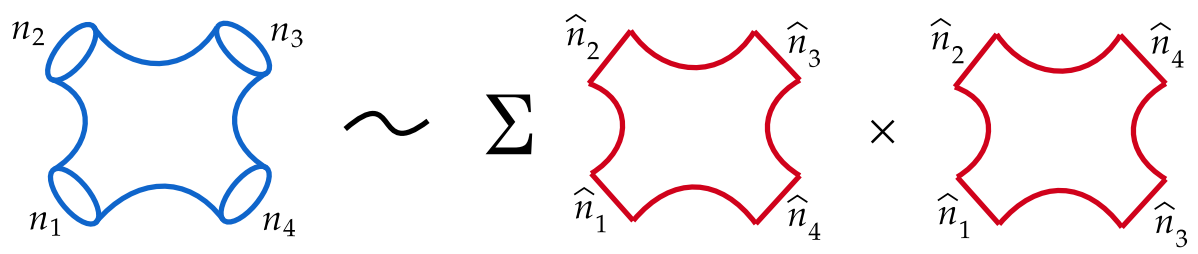

Figure 4. Four-point scattering amplitude of massive KK closed-strings with $\mathbb{Z}_{2}$-even parity (marked in blue) can be decomposed into a sum of the products of two color-ordered amplitudes of massive KK open-strings under the $\mathbb{S}^{1}$ compactification of $26 \mathrm{~d}$ for bosonic strings.

\section{KK graviton amplitudes from extended massive double-copy}

According to the extended massive KLT-like relation (2.22a), we can construct explicitly the four-point massive KK closed-string amplitude from the product of the corresponding massive KK open-string amplitudes as follows:

$$
\mathcal{A}_{\mathrm{cl}}(\zeta, k)=\frac{8 \kappa^{2}}{\pi \alpha^{\prime 5}} \sum_{\left\{a_{j}, b_{j}\right\}} \sum_{\left\{\mathrm{sgn}_{j}\right\}} \widehat{\varrho}_{a b} \sin \left(\pi \alpha^{\prime} \hat{k}_{1} \cdot \hat{k}_{2}\right)\left\{\overline{\mathcal{A}}_{\mathrm{op}}\left[\zeta_{j}^{a_{j}}, \hat{k}_{j} \mid 1234\right] \times \overline{\mathcal{A}}_{\mathrm{op}}\left[\zeta_{j}^{b_{j}}, \hat{k}_{j} \mid 1243\right]\right\}
$$

where we have replaced the closed-string coupling by the relation $g_{\mathrm{cl}}=\kappa /\left(2 \pi \alpha^{\prime}\right)$. For the two massive KK open-string amplitudes inside $\{\cdots\}$, the Regge slope should be rescaled as $\alpha^{\prime} \rightarrow \alpha^{\prime} / 4$. This is equivalent to considering the $N$-point low energy field theory formula (2.27) and derive the four-longitudinal KK graviton amplitude for the case of $N=4$. We illustrate in figure 4 the extended massive KLT-like relation (4.1) between the four-point scattering amplitude of KK closed-strings and the products of two color-ordered scattering amplitudes of KK open-strings.

\subsection{Constructing elastic scattering amplitudes of four KK gravitons}

In this subsection, we construct the four-point elastic scattering amplitudes of KK gravitons by using the extended massive KLT-like relation (4.1) under the low energy field theory limit.

Taking the zero-slope limit $\alpha^{\prime} \rightarrow 0$ for the closed-string amplitude (4.1), we derive the four-longitudinal KK graviton scattering amplitude for the elastic channel $(n, n) \rightarrow(n, n)$. Thus, we can express the elastic amplitude of longitudinal KK gravitons as the sum of products of two color-ordered massive KK gauge boson amplitudes:

$$
\begin{aligned}
& \mathcal{M}\left[1_{L}^{n} 2_{L}^{n} 3_{L}^{n} 4_{L}^{n}\right]=\frac{\kappa^{2}}{32} \sum_{\left\{a_{j}, b_{j}\right\}} \sum_{\left\{\mathrm{sgn}_{j}\right\}} \widehat{\varrho}_{a b}\left(-\hat{k}_{1} \cdot \hat{k}_{2}\right) \mathcal{T}\left[\zeta_{j}^{a_{j}}, \hat{k}_{j} \mid 1234\right] \times \mathcal{T}\left[\zeta_{j}^{b_{j}}, \hat{k}_{j} \mid 1243\right] \\
&=\frac{\kappa^{2}}{32} \sum_{\left\{a_{j}, b_{j}\right\}} \widehat{\varrho}_{a b}\{(s-\left.4 M_{n}^{2}\right) \mathcal{T}_{a_{j}}\left[1^{+n} 2^{+n} 3^{-n} 4^{-n}\right] \mathcal{T}_{b_{j}}\left[1^{+n} 2^{+n} 4^{-n} 3^{-n}\right] \\
&+s \mathcal{T}_{a_{j}}\left[1^{+n} 2^{-n} 3^{+n} 4^{-n}\right] \mathcal{T}_{b_{j}}\left[1^{+n} 2^{-n} 4^{-n} 3^{+n}\right] \\
&\left.+s \mathcal{T}_{a_{j}}\left[1^{+n} 2^{-n} 3^{-n} 4^{+n}\right] \mathcal{T}_{b_{j}}\left[1^{+n} 2^{-n} 4^{+n} 3^{-n}\right]\right\}
\end{aligned}
$$


where in the above second equality we have defined the short-hand notation for the partial amplitudes as $\mathcal{T}_{a_{j}}\left[1^{+n} 2^{-n} 3^{+n} 4^{-n}\right]=\mathcal{T}\left[1_{a_{1}}^{+n} 2_{a_{2}}^{-n} 3_{a_{3}}^{+n} 4_{a_{4}}^{-n}\right]$, and so on. Note that a massive KK graviton in $4 \mathrm{~d}$ has 5 physical helicity states and their polarization tensors are given by

$$
\begin{aligned}
& \zeta_{ \pm 2}^{\mu \nu}=\zeta_{ \pm 1}^{\mu} \zeta_{ \pm 1}^{\nu}, \quad \zeta_{ \pm 1}^{\mu \nu}=\frac{1}{\sqrt{2}}\left(\zeta_{ \pm 1}^{\mu} \zeta_{L}^{\nu}+\zeta_{L}^{\mu} \zeta_{ \pm 1}^{\nu}\right) \\
& \zeta_{L}^{\mu \nu}=\frac{1}{\sqrt{6}}\left(\zeta_{+1}^{\mu} \zeta_{-1}^{\nu}+\zeta_{-1}^{\mu} \zeta_{+1}^{\nu}+2 \zeta_{L}^{\mu} \zeta_{L}^{\nu}\right)
\end{aligned}
$$

Thus, in eq. (4.2) the helicity indices of each partial amplitude are $\left\{a_{j}, b_{j}\right\}=\{ \pm 1, L\}$. For the graviton polarization tensor $\zeta_{ \pm 2}^{\mu \nu}$, its coefficient $\varrho_{a_{j} b_{j}}=\varrho_{ \pm 1, \pm 1}=1$; for the polarization tensor $\zeta_{ \pm 1}^{\mu \nu}$, its coefficient $\varrho_{a_{j} b_{j}}=\varrho_{ \pm 1, L}=\varrho_{L, \pm 1}=\frac{1}{\sqrt{2}}$; and for the polarization tensor $\zeta_{L}^{\mu \nu}$, its coefficient $\varrho_{a_{j} b_{j}}$ takes the following values:

$$
\varrho_{a_{j} b_{j}}=\left\{\begin{aligned}
\sqrt{\frac{1}{6}}, & \text { for }\left\{a_{j}, b_{j}\right\}=\{ \pm 1, \mp 1\} \\
\sqrt{\frac{2}{3}}, & \text { for }\left\{a_{j}, b_{j}\right\}=L \\
0, & \text { for others }
\end{aligned}\right.
$$

We note that the longitudinal graviton's polarization tensor (4.3b) includes 3 products of two massive spin-1 polarization vectors, among which the two products $\zeta_{\mu}^{+1} \zeta_{\nu}^{-1}$ and $\zeta_{\mu}^{-1} \zeta_{\nu}^{+1}$ arise from the transverse polarization vectors and are important for the successful construction of full massive graviton amplitudes.

Then, we can compute all the color-ordered four-point gauge boson amplitudes from eqs. (3.24) and (3.27) with the relevant external polarization vectors (A.5) and momenta (A.1). Using these amplitudes, we derive the four-point elastic longitudinal KK graviton scattering amplitude from the extended massive double-copy formula (4.2):

$$
\mathcal{M}\left[1_{L}^{n} 2_{L}^{n} 3_{L}^{n} 4_{L}^{n}\right]=-\frac{\kappa^{2} M_{n}^{2}\left(X_{0}+X_{2} c_{2 \theta}+X_{4} c_{4 \theta}+X_{6} c_{6 \theta}\right) \csc ^{2} \theta}{512 \bar{s}(\bar{s}-4)\left[\bar{s}^{2}-(\bar{s}-4)^{2} c_{2 \theta}+24 \bar{s}+16\right]},
$$

where the coefficients $\left\{X_{j}\right\}$ in the numerator are given by

$$
\begin{aligned}
& X_{0}=-2\left(255 \bar{s}^{5}+2824 \bar{s}^{4}-19936 \bar{s}^{3}+39936 \bar{s}^{2}-256 \bar{s}+14336\right), \\
& X_{2}=429 \bar{s}^{5}-10152 \bar{s}^{4}+30816 \bar{s}^{3}-27136 \bar{s}^{2}-49920 \bar{s}+34816, \\
& X_{4}=2\left(39 \bar{s}^{5}-312 \bar{s}^{4}-2784 \bar{s}^{3}-11264 \bar{s}^{2}+26368 \bar{s}-2048\right), \\
& X_{6}=3 \bar{s}^{5}+40 \bar{s}^{4}+416 \bar{s}^{3}-1536 \bar{s}^{2}-3328 \bar{s}-2048 .
\end{aligned}
$$

The above formulas take exactly the same form as eq. (F.3a) and eqs. (F.4a)-(F.4d) of ref. [32]. We can compare our above elastic longitudinal KK graviton amplitude with that obtained by the previous explicit lengthy Feynman diagram calculations [50]. It is truly impressive that we find full agreement between our eqs. (4.5)-(4.6) and the eq. (71) of ref. [50] after taking into account the notational difference. Hence, our string-based massive doublecopy construction does successfully predict the exact four-point elastic scattering amplitude of longitudinal KK gravitons at tree level. In the next subsection, we will further present 
our string-based massive double-copy constructions of the inelastic scattering amplitudes of massive KK gravitons.

We can further derive the following LO and NLO amplitudes of eq. (4.5) under high energy expansion:

$$
\begin{aligned}
\mathcal{M}_{0}\left[1_{L}^{n} 2_{L}^{n} 3_{L}^{n} 4_{L}^{n}\right] & =\frac{3 \kappa^{2}}{128} s\left(7+c_{2 \theta}\right)^{2} \csc ^{2} \theta \\
\delta \mathcal{M}\left[1_{L}^{n} 2_{L}^{n} 3_{L}^{n} 4_{L}^{n}\right] & =-\frac{\kappa^{2} M_{n}^{2}}{256}\left(1810+93 c_{2 \theta}+126 c_{4 \theta}+19 c_{6 \theta}\right) \csc ^{4} \theta .
\end{aligned}
$$

We stress that the string-based double-copy formula (4.2) for 4-point amplitudes or (2.27) for general $N$-point amplitudes gives an explicit prescription on how to practically construct the exact tree-level $K K$ graviton scattering amplitudes from the sum of the products of the corresponding KK gauge boson amplitudes in the compactified KK field theories. Hence, given our string-based double-copy formula (2.27) or (4.2), one can practically follow this explicit prescription to derive the full KK graviton amplitudes without relying on computing the original KK string amplitudes.

Some further remarks are in order. It is instructive to compare our above string-based massive double-copy construction (à la extended KLT-like relations) with the extended BCJ-type double-copy construction under high energy expansion as given by section 5 of ref. [32]. At the leading order (LO) of the high energy expansion, both the 4-point longitudinal KK gauge boson amplitude and KK graviton amplitude are mass-independent, which are of $\mathcal{O}\left(E^{0}\right)$ and $\mathcal{O}\left(E^{2}\right)$, respectively. We found [32] that the BCJ-type numerators in the LO gauge boson amplitude satisfy the kinematic Jacobi identity, so the extension from the conventional massless BCJ method can be realized directly. At the next-to-leading order (NLO) of the high energy expansion, the longitudinal KK gauge boson amplitude and KK graviton amplitude become mass-dependent, which are of $\mathcal{O}\left(M_{n}^{2} / E^{2}\right)$ and $\mathcal{O}\left(M_{n}^{2} E^{0}\right)$, respectively. We further found [32] that the NLO numerators of the KK gauge boson amplitude can obey the Jacobi identity (after the generalized gauge transformations) and the double-copied KK graviton amplitude at NLO can give the correct structure of the exact NLO KK graviton amplitude, but the numeric coefficients of the double-copied NLO amplitude still differ somewhat from that of the exact NLO amplitude. So the NLO double-copy construction needs to be modified [32]. We note that an important reason of this problem is because the 4-point elastic amplitude of longitudinal KK gauge bosons contains double-poles with one type of poles from exchanging the massless zero-mode and another type of mass-poles from exchanging the level- $(2 n)$ KK-mode which contributes to the mass-dependent NLO amplitude. This is beyond the conventional BCJ double-copy method [18-20], so the deviation from it is expected at the NLO and the modified BCJtype double-copy construction was given for the NLO KK amplitudes [32]. As another reason, we note that the polarization tensor $\zeta_{L}^{\mu \nu}$ of the (helicity-zero) longitudinal KK graviton in eq. (4.3b) contains the sum of three products of two gauge boson polarization vectors $\left(\zeta_{+1}^{\mu} \zeta_{-1}^{\nu}, \zeta_{-1}^{\mu} \zeta_{+1}^{\nu}, \zeta_{L}^{\mu} \zeta_{L}^{\nu}\right)$, while the simple double-copy by using the longitudinal KK gauge boson amplitude alone could only provide the polarization-vector product of $\zeta_{L}^{\mu} \zeta_{L}^{\nu}$, which does not include the other two products $\left(\zeta_{+1}^{\mu} \zeta_{-1}^{\nu}, \zeta_{-1}^{\mu} \zeta_{+1}^{\nu}\right)$ of $\zeta_{L}^{\mu \nu}$ as given by the 
spin-1 helicity combinations $(+1,-1)$ and $(-1,+1)$. However, the sum of all three helicity combinations $(+1-1,-1+1, L L)$ for each external longitudinal KK graviton state in our present string-based massive double-copy formula (4.2) is automatically built in from the beginning. Another key feature of our string-based construction (4.2) is that it intrinsically includes a set of KK gauge boson sub-amplitudes with different KK-number combinations as allowed by the condition (3.3) due to the original string compactification under $\mathbb{S}^{1}$ (without the orbifold $\mathbb{Z}_{2}$ ) in section 2.1. This feature is intrinsically built in for our stringbased double-copy formulation.

We further note that extended BCJ-type double-copy construction of ref. [32] does work elegantly for the LO amplitudes under the high energy expansion. This is highly nontrivial because the longitudinal KK graviton polarization tensor $\zeta_{L}^{\mu \nu}$ also contains additional products of transverse polarization vectors $\left(\zeta_{+1}^{\mu} \zeta_{-1}^{\nu}, \zeta_{-1}^{\mu} \zeta_{+1}^{\nu}\right)$ which can contribute to the LO KK graviton amplitudes of $\mathcal{O}\left(E^{2} M_{n}^{0}\right)$. Note that our extended massive double-copy of LO amplitudes is built upon the KK gauge boson equivalence theorem (KK GAET) [41, 45] which connects the LO longitudinal KK gauge boson amplitude $\mathcal{T}_{0}\left[4 A_{L}^{a n}\right]$ to its corresponding LO KK Goldstone amplitude $\widetilde{\mathcal{T}}_{0}\left[4 A_{5}^{\text {an }}\right]$ :

$$
\mathcal{T}_{0}\left[4 A_{L}^{a n}\right]=\widetilde{\mathcal{T}}_{0}\left[4 A_{5}^{a n}\right]=\mathcal{O}\left(E^{0} M_{n}^{0}\right),
$$

where we have introduced the short-hand notations $\mathcal{T}_{0}\left[4 A_{L}^{a n}\right]=\mathcal{T}_{0}\left[A_{L}^{a n} A_{L}^{b n} \rightarrow A_{L}^{c n} A_{L}^{d n}\right]$ and $\widetilde{\mathcal{T}}_{0}\left[4 A_{5}^{a n}\right]=\widetilde{\mathcal{T}}_{0}\left[A_{5}^{a n} A_{5}^{b n} \rightarrow A_{5}^{c n} A_{5}^{d n}\right]$. We note that the KK Goldstone boson $A_{5}^{a n}$ is just a scalar field without any polarization vector, and $A_{5}^{a n}$ becomes a massless physical scalar degree of freedom in the high energy limit. Hence, the double-copy construction of the gravitational KK Goldstone boson $h_{n}^{55}$-amplitude from the KK Goldstone $A_{n}^{a 5}$-amplitude is uniquely defined via the correspondence $A_{n}^{a 5} \otimes A_{n}^{a 5} \rightarrow h_{n}^{55}$. Hence, the LO gravitational KK Goldstone amplitude $\widetilde{\mathcal{M}}_{0}\left[4 h_{n}^{55}\right]$ as given by the double-copy of the LO KK Goldstone amplitude $\widetilde{\mathcal{T}}_{0}\left[4 A_{n}^{a 5}\right]$ is well defined under one-to-one correspondence. On the other hand, we established the Gravitational Equivalence Theorem (GRET) [32] which connects the LO longitudinal KK graviton amplitude $\mathcal{M}_{0}\left[4 h_{L}^{n}\right]$ to its corresponding LO gravitational KK Goldstone amplitude $\widetilde{\mathcal{M}}_{0}\left[4 h_{55}^{n}\right]$ :

$$
\mathcal{M}_{0}\left[4 h_{L}^{n}\right]=\widetilde{\mathcal{M}}_{0}\left[4 h_{55}^{n}\right]=\mathcal{O}\left(E^{2} M_{n}^{0}\right),
$$

where we have introduced the short-hand notations $\mathcal{M}_{0}\left[4 h_{L}^{n}\right]=\mathcal{M}_{0}\left[h_{L}^{n} h_{L}^{n} \rightarrow h_{L}^{n} h_{L}^{n}\right]$ and $\widetilde{\mathcal{M}}_{0}\left[4 h_{55}^{n}\right]=\mathcal{M}_{0}\left[h_{55}^{n} h_{55}^{n} \rightarrow h_{55}^{n} h_{55}^{n}\right]$. Thus, the LO equality of the KK GAET (4.8) leads to the LO equality of the KK GRET (4.9) by the double-copy construction. Hence, the success of our extended double-copy construction of the LO longitudinal KK graviton amplitude $\mathcal{M}_{0}\left[4 h_{L}^{n}\right]$ from the LO longitudinal $K K$ gauge boson amplitude $\mathcal{T}_{0}\left[4 A_{L}^{a n}\right]$ is fully ensured by the correct double-copy construction of its corresponding LO gravitational KK Goldstone amplitude $\widetilde{\mathcal{M}}_{0}\left[4 h_{n}^{55}\right]$ from the LO gauge $K K$ Goldstone amplitude $\widetilde{\mathcal{T}}_{0}\left[4 A_{n}^{a 5}\right]$ because we can derive the correspondence GAET $\Longrightarrow$ GRET by using the LO double-copy construction. This means that we can correctly compute the LO longitudinal KK graviton amplitude $\mathcal{M}_{0}\left[4 h_{L}^{n}\right]$ by defining an effective LO polarization tensor for each longitudinal KK graviton:

$$
\zeta_{L(0)}^{\mu \nu}=\zeta_{L}^{\mu} \zeta_{L}^{\nu}
$$


We have verified this insight by explicitly computing the four-point elastic scattering amplitude $\mathcal{M}_{0}\left[4 h_{L}^{n}\right]$ of longitudinal KK gravitons with the LO polarization tensor (4.10). Namely, using the LO longitudinal polarization tensor (4.10) can give the same LO longitudinal graviton amplitude as that of the full longitudinal polarization tensor $(4.3 \mathrm{~b})$. The difference between the longitudinal KK graviton amplitudes as computed by using the two types of polarization tensors (4.3b) and (4.10) belongs to the mass-dependent residual term of the GRET [32],

$$
\mathcal{M}_{\Delta}=\delta \mathcal{M}\left[4 h_{L}^{n}\right]-\delta \widetilde{\mathcal{M}}\left[4 h_{55}^{n}\right]=\mathcal{O}\left(E^{0} M_{n}^{2}\right),
$$

where $\mathcal{M}=\mathcal{M}_{0}+\delta \mathcal{M}$ and $\widetilde{\mathcal{M}}=\widetilde{\mathcal{M}}_{0}+\delta \widetilde{\mathcal{M}}$ under the high energy expansion. The residual term (4.11) is derived from the GRET identity [32], $\mathcal{M}\left[4 h_{L}^{n}\right]=\widetilde{\mathcal{M}}\left[4 h_{55}^{n}\right]+\mathcal{M}_{\Delta}$, together with the LO GRET equality (4.9).

\subsection{Constructing inelastic scattering amplitudes of four KK gravitons}

In this subsection, we study the four-point inelastic scattering channels $(n, n) \rightarrow(m, m)$ and $(0,0) \rightarrow(n, n)$ for the massive KK closed-string amplitudes (4.1). We will take the low energy field theory limit $\alpha^{\prime} \rightarrow 0$ and derive the inelastic scattering amplitudes of fourlongitudinal KK gravitons.

For inelastic scattering channels $(n, n) \rightarrow(m, m)$, we obtain the following extended massive KLT-like four-point formula, which expresses the inelastic scattering amplitude of longitudinal KK gravitons as the sum of products of the corresponding color-ordered KK gauge boson scattering amplitudes:

$$
\begin{aligned}
\mathcal{M}\left[1_{L}^{n} 2_{L}^{n} 3_{L}^{m} 4_{L}^{m}\right]=\frac{\kappa^{2}}{32} \sum_{\left\{a_{j}, b_{j}\right\}} \widehat{\varrho}_{a b}\{ & s \mathcal{T}_{a_{j}}\left[1^{+n} 2^{-n} 3^{+m} 4^{-m}\right] \mathcal{T}_{b_{j}}\left[1^{+n} 2^{-n} 4^{-m} 3^{+m}\right] \\
& \left.+s \mathcal{T}_{a_{j}}\left[1^{+n} 2^{-n} 3^{-m} 4^{+m}\right] \mathcal{T}_{b_{j}}\left[1^{+n} 2^{-n} 4^{+m} 3^{-m}\right]\right\} .
\end{aligned}
$$

Then, using the color-ordered inelastic KK gauge boson scattering amplitudes in section 3.1 and our extended massive double-copy formula (4.12), we construct the four-point inelastic longitudinal KK graviton scattering amplitude of $(n, n) \rightarrow(m, m)$, which takes the following compact form:

$$
\mathcal{M}\left[1_{L}^{n} 2_{L}^{n} 3_{L}^{m} 4_{L}^{m}\right]=-\frac{\kappa^{2} M_{n}^{2}\left(X_{0}+X_{1} c_{\theta}+X_{2} c_{2 \theta}\right)\left(Y_{0}+Y_{2} c_{2 \theta}+Y_{4} c_{4 \theta}+Y_{6} c_{6 \theta}\right)}{1024 \bar{s}\left[\left(\bar{s}-4 \bar{q} \bar{q}^{\prime} c_{\theta}\right)^{2}+16 r^{2}\right]^{2}\left[\left(\bar{s}+4 \bar{q} \bar{q}^{\prime} c_{\theta}\right)^{2}-16 r^{2}\right]},
$$

where the coefficients $\left(X_{j}, Y_{j}\right)$ in the numerator are polynomial functions of the scattering energy and momenta,

$$
\begin{aligned}
X_{0}= & 3 \bar{s}^{2}-4 r_{+}^{2} \bar{s}-16 r^{2}, \quad X_{1}=-16 \bar{q} \bar{q}^{\prime} \bar{s}, \quad X_{2}=16 \bar{q}^{2} \bar{q}^{2}, \\
Y_{0}= & -170 \bar{s}^{6}-84 r_{+}^{2} \bar{s}^{5}+32\left(39 r^{4}+135 r^{2}+39\right) \bar{s}-128\left(17 r^{6}+69 r^{4}+69 r^{2}+17\right) \bar{s}^{3} \\
& +512\left(39 r^{4}+51 r^{2}+39\right) r^{2} \bar{s}^{2}-51200\left(r^{2}+1\right) \bar{s}+57344 r^{6}, \\
Y_{2}= & 143 \bar{s}^{6}-1804 r_{+}^{2} \bar{s}^{5}+16\left(227 r^{4}+391 r^{2}+227\right) \bar{s}^{4} \\
& -64\left(15 r^{6}-121 r^{4}-121 r^{2}+15\right) \bar{s}^{3}-256\left(61 r^{4}+345 r^{2}+61\right) r^{2} \bar{s}^{2} \\
& +95232 r_{+}^{2} r^{4} \bar{s}-69632 r^{6}, \\
Y_{4}= & 2\left[13 \bar{s}^{6}-44 r_{+}^{2} \bar{s}^{5}-16\left(23 r^{4}+79 r^{2}+23\right) \bar{s}^{4}+64\left(9 r^{6}+29 r^{4}+29 r^{2}+9\right) \bar{s}^{3}\right.
\end{aligned}
$$




$$
\begin{aligned}
& \left.+256\left(25 r^{4}+53 r^{2}+25\right) r^{2} \bar{s}^{2}-23552 r_{+}^{2} r^{4} \bar{s}+4096 r^{6}\right], \\
Y_{6}= & \left(\bar{s}^{3}+12 r^{2} \bar{s}^{2}-48 r^{4} \bar{s}-64 r^{6}\right)\left(\bar{s}^{3}+12 \bar{s}^{2}-48 \bar{s}-64\right),
\end{aligned}
$$

where $\bar{s}=s / M_{n}^{2}, r=M_{m} / M_{n}$ and $r_{+}^{2}=1+r^{2}$. From the above eq. (4.13), we can further derive the LO and NLO scattering amplitudes of the KK gravitons under high energy expansion:

$$
\begin{aligned}
& \mathcal{M}_{0}\left[1_{L}^{n} 2_{L}^{n} 3_{L}^{m} 4_{L}^{m}\right]=\frac{\kappa^{2}}{64} s\left(7+c_{2 \theta}\right)^{2} \csc ^{2} \theta=\frac{2}{3} \mathcal{M}_{0}\left[1_{L}^{n} 2_{L}^{n} 3_{L}^{n} 4_{L}^{n}\right], \\
& \delta \mathcal{M}\left[1_{L}^{n} 2_{L}^{n} 3_{L}^{m} 4_{L}^{m}\right]=-\frac{\kappa^{2} M_{n}^{2}}{128}\left(1+r^{2}\right)\left(410+59 c_{2 \theta}+38 c_{4 \theta}+5 c_{6 \theta}\right) \csc ^{4} \theta .
\end{aligned}
$$

We note that the eq. (4.15a) just equals $\frac{2}{3}$ times the LO elastic KK graviton amplitude (4.7a). This factor $\frac{2}{3}$ can be understood in a transparent way from our string-based double-copy formulation. The reason is that the elastic scattering $(n, n) \rightarrow(n, n)$ contains 6 sub-amplitudes (figure 1) and the inelastic scattering $(n, n) \rightarrow(m, m)$ includes only 4 sub-amplitudes (figure 2). These sub-amplitude take the same LO form under the high energy limit which is mass-independent. Hence, we deduce the connection factor $\frac{4}{6}=\frac{2}{3}$ between the two LO amplitudes.

Next, we analyze the mixed inelastic channel of KK gauge boson scattering $(0,0) \rightarrow$ $(n, n)$. We find that the condition (2.18) allows only two combinations of the external KK states (as originally defined under the $\mathbb{S}^{1}$ compactification of $26 \mathrm{~d}$ ), $\{0,0,+n,-n\}$ and $\{0,0,-n,+n\}$. Thus, from eq. (4.1), we derive the following extended four-point massive KK double-copy formula which expresses the inelastic amplitude of longitudinal KK gravitons as the sum of products of the corresponding color-ordered KK gauge boson amplitudes:

$$
\mathcal{M}\left[1_{T}^{0} 2_{T}^{0} 3_{L}^{n} 4_{L}^{n}\right]=\frac{\kappa^{2}}{32} \sum_{\left\{a_{j}, b_{j}\right\}}\left(\widehat{\varrho}_{a b} s \mathcal{T}_{a_{j}}\left[1^{0} 2^{0} 3^{+n} 4^{-n}\right] \mathcal{T}_{b_{j}}\left[1^{0} 2^{0} 4^{-n} 3^{+n}\right]\right)
$$

where in the amplitude $\mathcal{M}\left[1_{T}^{0} 2_{T}^{0} 3_{L}^{n} 4_{L}^{n}\right]$ the subscript $T= \pm 2$ denotes the helicities of the massless zero-mode gravitons in the initial state.

With the above and using our extended massive double-copy formula (4.16), we construct the mixed four-point inelastic graviton amplitudes of $(0,0) \rightarrow(n, n)$ and derive following form:

$$
\mathcal{M}\left[1_{ \pm 2}^{0} 2_{\mp 2}^{0} 3_{L}^{n} 4_{L}^{n}\right]=\frac{\kappa^{2} M_{n}^{2}\left(\bar{s}^{2}+16 \bar{s}+16\right) s_{\theta}^{4}}{16\left[(\bar{s}+4)-(\bar{s}-4) c_{2 \theta}\right]} .
$$

We can further derive the following LO and NLO amplitudes from eq. (4.17) under high energy expansion:

$$
\begin{aligned}
\mathcal{M}_{0}\left[1_{ \pm 2}^{0} 2_{\mp 2}^{0} 3_{L}^{n} 4_{L}^{n}\right] & =\frac{\kappa^{2}}{32} s s_{\theta}^{2}, \\
\delta \mathcal{M}\left[1_{ \pm 2}^{0} 2_{\mp 2}^{0} 3_{L}^{n} 4_{L}^{n}\right] & =\frac{\kappa^{2} M_{n}^{2}}{16}\left(3-5 c_{2 \theta}\right) .
\end{aligned}
$$




\subsection{Constructing multi-point scattering amplitudes of KK gravitons}

Our above analyses of the four-point scattering amplitudes can be further extended to the $N$-point amplitudes with $N \geqslant 5$. We first consider a five-point inelastic scattering process $(2 n, n) \rightarrow(n, n, n)$, where all the external KK states are set to be $\mathbb{Z}_{2}$ even. We find that the condition (2.18) of KK number conservation allows the following combinations of the KK numbers of the external-state gauge bosons for the sub-amplitudes:

$$
\begin{array}{ll}
\{+2 n,+n,-n,-n,-n\}, & \{+2 n,-n,+n,-n,-n\}, \quad\{+2 n,-n,-n,+n,-n\}, \\
\{+2 n,-n,-n,-n,+n\}, & \{\text { all permutations of }(+,-)\}
\end{array}
$$

where we assign the KK number of particle- 1 as $+2 n$ for convenience.

Thus, using the general formula (2.27) of the $N$-point KK graviton amplitudes, we derive the five-point longitudinal KK graviton scattering amplitude as follows:

$$
\begin{aligned}
& \mathcal{M}\left[1_{L}^{2 n} 2_{L}^{n} 3_{L}^{n} 4_{L}^{n} 5_{L}^{n}\right]=\frac{\kappa^{3}}{128 \sqrt{2}} \sum_{\left\{a_{j}, b_{j}\right\}} \widehat{\varrho}_{a b}\{ \\
& \left\{\left(s_{12}-9 M_{n}^{2}\right)\left(s_{13}-M_{n}^{2}\right)\right. \\
& \times \mathcal{T}_{a_{j}}\left[1^{+2 n} 2^{+n} 3^{-n} 4^{-n} 5^{-n}\right] \mathcal{T}_{b_{j}}\left[1^{+2 n} 3^{-n} 2^{+n} 5^{-n} 4^{-n}\right] \\
& +\left(s_{12}+s_{23}-9 M_{n}^{2}\right)\left(s_{13}-M_{n}^{2}\right) \\
& \left.\times \mathcal{T}_{a_{j}}\left[1^{+2 n} 2^{+n} 3^{-n} 4^{-n} 5^{-n}\right] \mathcal{T}_{b_{j}}\left[1^{+2 n} 2^{+n} 3^{-n} 5^{-n} 4^{-n}\right]\right\} \\
& +\left\{\left(s_{12}-M_{n}^{2}\right)\left(s_{13}-9 M_{n}^{2}\right)\right. \\
& \times \mathcal{T}_{a_{j}}\left[1^{+2 n} 2^{-n} 3^{+n} 4^{-n} 5^{-n}\right] \mathcal{T}_{b_{j}}\left[1^{+2 n} 3^{+n} 2^{-n} 5^{-n} 4^{-n}\right] \\
& +\left(s_{12}+s_{23}-M_{n}^{2}\right)\left(s_{13}-9 M_{n}^{2}\right) \\
& \left.\times \mathcal{T}_{a_{j}}\left[1^{+2 n} 2^{-n} 3^{+n} 4^{-n} 5^{-n}\right] \mathcal{T}_{b_{j}}\left[1^{+2 n} 2^{-n} 3^{+n} 5^{-n} 4^{-n}\right]\right\} \\
& +\left\{\left(s_{12}-M_{n}^{2}\right)\left(s_{13}-M_{n}^{2}\right)\right. \\
& \times \mathcal{T}_{a_{j}}\left[1^{+2 n} 2^{-n} 3^{-n} 4^{+n} 5^{-n}\right] \mathcal{T}_{b_{j}}\left[1^{+2 n} 3^{-n} 2^{-n} 5^{-n} 4^{+n}\right] \\
& +\left(s_{12}+s_{23}-5 M_{n}^{2}\right)\left(s_{13}-M_{n}^{2}\right) \\
& \left.\times \mathcal{T}_{a_{j}}\left[1^{+2 n} 2^{-n} 3^{-n} 4^{+n} 5^{-n}\right] \mathcal{T}_{b_{j}}\left[1^{+2 n} 2^{-n} 3^{-n} 5^{-n} 4^{+n}\right]\right\} \\
& +\left\{\left(s_{12}-M_{n}^{2}\right)\left(s_{13}-M_{n}^{2}\right)\right. \\
& \times \mathcal{T}_{a_{j}}\left[1^{+2 n} 2^{-n} 3^{-n} 4^{-n} 5^{+n}\right] \mathcal{T}_{b_{j}}\left[1^{+2 n} 3^{-n} 2^{-n} 5^{+n} 4^{-n}\right] \\
& +\left(s_{12}+s_{23}-5 M_{n}^{2}\right)\left(s_{13}-M_{n}^{2}\right) \\
& \left.\times \mathcal{T}_{a_{j}}\left[1^{+2 n} 2^{-n} 3^{-n} 4^{-n} 5^{+n}\right] \mathcal{T}_{b_{j}}\left[1^{+2 n} 2^{-n} 3^{-n} 5^{+n} 4^{-n}\right]\right\} \\
& + \text { all permutations of }(+n,-n)\} \text {. }
\end{aligned}
$$

Then, we consider the six-point scattering process with all the external states being $\mathbb{Z}_{2}$ even. The KK numbers of the six external states are chosen as $\left(\hat{n}_{1}, \hat{n}_{2}, \hat{n}_{3}, \hat{n}_{4}, \hat{n}_{5}, \hat{n}_{6}\right)=$ $(n, n, n, n, n, n)$. From the condition (2.18) of KK number conservation, we deduce the following allowed combinations of the KK numbers of the external-state gauge bosons of 
the sub-amplitudes:

$$
\begin{aligned}
& \{+n,+n,+n,-n,-n,-n\},\{+n,+n,-n,+n,-n,-n\}, \\
& \{+n,+n,-n,-n,+n,-n\},\{+n,+n,-n,-n,-n,+n\}, \\
& \{+n,-n,+n,+n,-n,-n\},\{+n,-n,+n,-n,+n,-n\}, \\
& \{+n,-n,+n,-n,-n,+n\},\{+n,-n,-n,+n,+n,-n\}, \\
& \{+n,-n,-n,+n,-n,+n\},\{+n,-n,-n,-n,+n,+n\}, \\
& \text { all permutations of }(+,-)\} .
\end{aligned}
$$

Using the above decomposition and eq. (2.27) of the general $N$-point KK graviton amplitudes, we obtain the six-point longitudinal KK graviton scattering amplitude as follows:

$$
\begin{aligned}
& \mathcal{M}\left[1_{L}^{n} 2_{L}^{n} 3_{L}^{n} 4_{L}^{n} 5_{L}^{n} 6_{L}^{n}\right]=\frac{\kappa^{4}}{1024} \sum_{\left\{a_{j}, b_{j}\right\}} \widehat{\varrho}_{a b}\{ \\
& s_{14}\left\{\left(s_{12}-4 M_{n}^{2}\right)\left(s_{13}-4 M_{n}^{2}\right)\right. \\
& \times \mathcal{T}_{a_{j}}\left[1^{+n} 2^{+n} 3^{+n} 4^{-n} 5^{-n} 6^{-n}\right] \mathcal{T}_{b_{j}}\left[1^{+n} 4^{-n} 3^{+n} 2^{+n} 6^{-n} 5^{-n}\right] \\
& +\left(s_{12}-4 M_{n}^{2}\right)\left(s_{13}+s_{34}-4 M_{n}^{2}\right) \\
& \times \mathcal{T}_{a_{j}}\left[1^{+n} 2^{+n} 3^{+n} 4^{-n} 5^{-n} 6^{-n}\right] \mathcal{T}_{b_{j}}\left[1^{+n} 3^{+n} 4^{-n} 2^{+n} 6^{-n} 5^{-n}\right] \\
& +\left(s_{13}-4 M_{n}^{2}\right)\left(s_{12}+s_{23}-8 M_{n}^{2}\right) \\
& \times \mathcal{T}_{a_{j}}\left[1^{+n} 2^{+n} 3^{+n} 4^{-n} 5^{-n} 6^{-n}\right] \mathcal{T}_{b_{j}}\left[1^{+n} 4^{-n} 2^{+n} 3^{+n} 6^{-n} 5^{-n}\right] \\
& -\left(s_{25}+s_{26}\right)\left(s_{13}-4 M_{n}^{2}\right) \\
& \times \mathcal{T}_{a_{j}}\left[1^{+n} 2^{+n} 3^{+n} 4^{-n} 5^{-n} 6^{-n}\right] \mathcal{T}_{b_{j}}\left[1^{+n} 2^{+n} 4^{-n} 3^{+n} 6^{-n} 5^{-n}\right] \\
& +\left(s_{12}+s_{24}-4 M_{n}^{2}\right)\left(s_{13}+s_{34}-4 M_{n}^{2}\right) \\
& \times \mathcal{T}_{a_{j}}\left[1^{+n} 2^{+n} 3^{+n} 4^{-n} 5^{-n} 6^{-n}\right] \mathcal{T}_{b_{j}}\left[1^{+n} 3^{+n} 2^{+n} 4^{-n} 6^{-n} 5^{-n}\right] \\
& -\left(s_{25}+s_{26}\right)\left(s_{13}+s_{34}-4 M_{n}^{2}\right) \\
& \left.\times \mathcal{T}_{a_{j}}\left[1^{+n} 2^{+n} 3^{+n} 4^{-n} 5^{-n} 6^{-n}\right] \mathcal{T}_{b_{j}}\left[1^{+n} 2^{+n} 3^{+n} 4^{-n} 6^{-n} 5^{-n}\right]\right\} \\
& + \text { all permutations of }(+n,-n)\} \text {. }
\end{aligned}
$$

The above five-point and six-point KK gauge boson amplitudes and KK graviton amplitudes are worth of further systematic studies and we will pursue these in the future works.

\section{Conclusions}

The Kaluza-Klein (KK) compactification [1,2] of higher dimensional spacetime is a fundamental ingredient of the major directions for the new physics beyond the standard model (SM), including the string/M theories $[3,4]$ and extra dimensional field theories with large or small extra dimensions [5-9]. Studying the double-copy construction of graviton scattering amplitudes from gauge boson scattering amplitudes has pointed to profound deep connections between the gauge forces and gravitational force in nature.

So far substantial efforts have been made to formulate and test the double-copy constructions between the massless gauge theories and massless general relativity (GR) [20]. 
But the extensions of conventional double-copy method to massive gauge/gravity theories are generally difficult, because most of such theories (including the massive Yang-Mills theory and massive Fierz-Pauli gravity) violate explicitly the gauge symmetry and diffeomorphism invariance (which are the key for successful double-copy construction). The two important candidates with promise include the compactified KK gauge/gravity theories and the topologically massive Chern-Simons gauge/gravity theories. The extended BCJ-type double-copy construction for realistic massive KK gauge/gravity theories was found [32] to be highly nontrivial even for the four-point KK scattering amplitudes at tree level, and proper modifications of the conventional BCJ method are generally needed for the KK scattering amplitudes at the next-to-leading order (NLO) of the high energy expansion [32].

In this work, we studied the scattering amplitudes of massive KK states of open and closed bosonic strings under toroidal compactification. The essential advantage of the compactified KK string theory is that the connection between the KK closed-string amplitudes and the proper products of KK open-string amplitudes can be intrinsically built in from the start. For the present study, we take bosonic string as a computational tool for establishing the massive KLT-like relations of KK string states and for deriving the low energy KK graviton scattering amplitudes.

In section 2.1, we set up the toroidal compactification for the $26 \mathrm{~d}$ bosonic string theory where the 21 of the extra spatial dimensions have very small compactification radii of $\mathcal{O}\left(M_{\mathrm{Pl}}^{-1}\right)$ and get decoupled in our effective string theory below the Planck scale. Thus, we only deal with the KK strings in a single compactified 25 th spatial dimension under $\mathbb{S}^{1}$ with relatively larger radius $R$. With these, in section 2.2 we studied vertex operators of the KK open and closed strings. In particular, these include a class of vertex operators having $\mathbb{Z}_{2}$-even parity whose scattering amplitudes will give, in the field-theory limit, the corresponding scattering amplitudes of the KK gauge bosons and of the KK gravitons in the KK gauge/gravity theories under the $5 \mathrm{~d}$ compactification of $\mathbb{S}^{1} / \mathbb{Z}_{2}$. Then, we formulated the $N$-point scattering amplitudes (2.22) of the KK open and closed strings in section 2.3, and further derived the corresponding scattering amplitudes (2.27) of the KK gauge bosons and KK gravitons in the low energy field-theory limit in section 2.4. We observed that any KK amplitude with external states being $\mathbb{Z}_{2}$ even (odd) should be decomposed into a sum of relevant sub-amplitudes whose external states have KK numbers obey the conservation condition (2.18).

In section 3 , using the formulas of section 2 we computed explicitly the four-point color-ordered scattering amplitudes of KK open strings and derived the corresponding gauge boson scattering amplitudes in the field theory limit. The four-point elastic KK gauge boson scattering amplitudes are given in eqs. (3.4)-(3.7) of section 3.1, while the four-point inelastic KK gauge boson scattering amplitudes are given in eqs. (3.10)-(3.12) and eq. (3.17) of section 3.2. Then, in section 3.3, we analyzed the structure of the colorordered scattering amplitudes of massive KK gauge bosons. We demonstrated that the tree-level massive KK gauge boson amplitudes can be obtained from the corresponding color-ordered amplitudes of the massless zero-mode gauge bosons by making proper shifts of the Mandelstam variables. This serves as an elegant and efficient method to compute any color-ordered massive KK gauge boson amplitudes. 
In section 4, we applied the massive KLT-like relation (4.1) of four-point KK string amplitudes by taking the field theory limit, and derived the double-copy formulas (4.2), (4.12) and (4.16) for constructing the four-point KK graviton scattering amplitudes. These give an explicit prescription on how to construct the exact four-point KK graviton amplitudes from the sum of relevant products of the corresponding color-ordered KK gauge boson amplitudes. With these, we computed the exact tree-level four-point elastic KK graviton scattering amplitudes (4.5)-(4.6) in section 4.1, and the exact tree-level four-point inelastic KK graviton scattering amplitudes (4.13)-(4.14) and (4.17) in section 4.2. Finally, in section 4.3, we used our general string-based double-copy construction formula (2.27) to obtain the five-point and six-point scattering amplitudes of massive KK gravitons as given by eq. (4.20) and eq. (4.22).

\section{Acknowledgments}

We thank Henry Tye for valuable discussions. We also thank Ziqi Yan for discussing ref. [48]. The works of HJH, YFH and YL were supported in part by National NSF of China (under grants Nos.11835005, 12175136), by National Key R\&D Program of China (under grant No.2017YFA0402204), and by the CAS Center for Excellence in Particle Physics (CCEPP). The research of SH was supported in part by National Natural Science Foundation of China (under Grant Nos.11935013, 11947301, 12047502, 12047503).

\section{A Kinematics of four-point scattering amplitudes of KK states}

In this appendix we present the kinematics of four-particle scattering processes of KK states in the $(3+1) \mathrm{d}$ spacetime. We choose the Minkowski metric tensor $\eta^{\mu \nu}=\eta_{\mu \nu}=$ $\operatorname{diag}(-1,1,1,1)$.

For the four-particle elastic scattering of KK states $(n, n) \rightarrow(n, n)$, we define the 4momenta of the external KK states in the center-of-mass frame as follows:

$$
\begin{array}{ll}
p_{1}^{\mu}=-E(1,0,0, \beta), & p_{2}^{\mu}=-E(1,0,0,-\beta), \\
p_{3}^{\mu}=E\left(1, \beta s_{\theta}, 0, \beta c_{\theta}\right), & p_{4}^{\mu}=E\left(1,-\beta s_{\theta}, 0,-\beta c_{\theta}\right),
\end{array}
$$

where $\left(s_{\theta}, c_{\theta}\right)=(\sin \theta, \cos \theta)$ and $\beta=\left(1-M_{n}^{2} / E^{2}\right)^{1 / 2}$. With the above, we define the three Mandelstam variables:

$$
\begin{aligned}
& s=-\left(p_{1}+p_{2}\right)^{2}=4 E^{2}, \\
& t=-\left(p_{1}+p_{4}\right)^{2}=-\frac{1}{2} s \beta^{2}\left(1+c_{\theta}\right), \\
& u=-\left(p_{1}+p_{3}\right)^{2}=-\frac{1}{2} s \beta^{2}\left(1-c_{\theta}\right),
\end{aligned}
$$

where $\beta=\left(1-M_{n}^{2} / E^{2}\right)^{1 / 2}$. With the on-shell condition $E^{2}=E^{2} \beta^{2}+M_{n}^{2}$, we may define the following set of Mandelstam variables:

$$
s_{0}=4 E^{2} \beta^{2}, \quad t_{0}=-\frac{1}{2} s_{0}\left(1+c_{\theta}\right), \quad u_{0}=-\frac{1}{2} s_{0}\left(1-c_{\theta}\right),
$$


where we have introduced the notation $s_{0}=s-4 M_{n}^{2}$, and thus $\left(s_{0}, t_{0}, u_{0}\right)=\left(s \beta^{2}, t, u\right)$. Summing up the Mandelstam variables (A.2) and (A.3) gives the following relations:

$$
s+t+u=4 M_{n}^{2}, \quad s_{0}+t_{0}+u_{0}=0 .
$$

Then, we define the following polarization vectors for the external KK gauge bosons in the center-of-mass frame:

$$
\begin{aligned}
\zeta_{1, \pm 1}^{\mu} & =\frac{1}{\sqrt{2}}(0,1, \pm \mathrm{i}, 0), & \zeta_{1, L}^{\mu} & =\frac{E}{M_{n}}(\beta, 0,0,1), \\
\zeta_{2, \pm 1}^{\mu} & =\frac{1}{\sqrt{2}}(0,1, \mp \mathrm{i}, 0), & \zeta_{2, L}^{\mu} & =\frac{E}{M_{n}}(\beta, 0,0,-1), \\
\zeta_{3, \pm 1}^{\mu} & =\frac{1}{\sqrt{2}}\left(0, \mp \mathrm{i} c_{\theta}, 1, \pm \mathrm{i} s_{\theta}\right), & \zeta_{3, L}^{\mu} & =\frac{E}{M_{n}}\left(\beta, s_{\theta}, 0, c_{\theta}\right), \\
\zeta_{4, \pm 1}^{\mu} & =\frac{1}{\sqrt{2}}\left(0, \pm \mathrm{i} c_{\theta}, 1, \mp \mathrm{i} s_{\theta}\right), & \zeta_{4, L}^{\mu} & =\frac{E}{M_{n}}\left(\beta,-s_{\theta}, 0,-c_{\theta}\right) .
\end{aligned}
$$

Next, we consider the inelastic KK scattering process of $(n, n) \rightarrow(m, m)$. Thus, the 4-momenta of the external states in the center-of-mass frame can be defined as follows:

$$
\begin{array}{ll}
p_{1}^{\mu}=-E(1,0,0, \beta), & p_{2}^{\mu}=-E(1,0,0,-\beta), \\
p_{3}^{\mu}=E\left(1, \beta^{\prime} s_{\theta}, 0, \beta^{\prime} c_{\theta}\right), & p_{4}^{\mu}=E\left(1,-\beta^{\prime} s_{\theta}, 0,-\beta^{\prime} c_{\theta}\right),
\end{array}
$$

where $\beta=\left(1-M_{n}^{2} / E^{2}\right)^{1 / 2}$ and $\beta^{\prime}=\left(1-M_{m}^{2} / E^{2}\right)^{1 / 2}$. With these, we define the Mandelstam variables:

$$
\begin{aligned}
& s=-\left(p_{1}+p_{2}\right)^{2}=4 E^{2}, \\
& t=-\left(p_{1}+p_{4}\right)^{2}=-\frac{1}{4} s\left(\beta^{2}+\beta^{2}+2 \beta \beta^{\prime} c_{\theta}\right), \\
& u=-\left(p_{1}+p_{3}\right)^{2}=-\frac{1}{4} s\left(\beta^{2}+\beta^{2}-2 \beta \beta^{\prime} c_{\theta}\right),
\end{aligned}
$$

from which we have $s+t+u=2\left(M_{n}^{2}+M_{m}^{2}\right)$. The corresponding longitudinal polarization vectors of the $\mathrm{KK}$ gauge bosons are given by

$$
\begin{aligned}
\zeta_{1, L}^{\mu} & =\frac{E}{M_{n}}(\beta, 0,0,1), & \zeta_{2, L}^{\mu} & =\frac{E}{M_{n}}(\beta, 0,0,-1), \\
\zeta_{3, L}^{\mu} & =\frac{E}{M_{m}}\left(\beta^{\prime}, s_{\theta}, 0, c_{\theta}\right), & \zeta_{4, L}^{\mu} & =\frac{E}{M_{m}}\left(\beta^{\prime},-s_{\theta}, 0,-c_{\theta}\right),
\end{aligned}
$$

while their transverse polarization vectors are mass-independent and remain the same as in eq. (A.5). For another inelastic scattering channel $(0,0) \rightarrow(m, m)$, we just set the initial state masses to be zero $\left(M_{n} \rightarrow 0\right)$ in the above setup and only allow transverse polarizations for the massless initial state gauge bosons.

\section{B Full scattering amplitudes of KK gauge and Goldstone bosons}

In this Appendix, for the sake of comparison we present systematically the four-point elastic and inelastic scattering amplitudes of KK gauge bosons in the 5d KK Yang-Mills gauge theories under the orbifold compactification of $\mathbb{S}^{1} / \mathbb{Z}_{2}$. 


\section{B.1 Elastic KK gauge and Goldstone boson scattering amplitudes}

According to ref. [32], we summarize the four-point elastic scattering amplitudes of longitudinal KK gauge bosons and of KK Goldstone bosons as follows:

$$
\begin{aligned}
& \mathcal{T}\left[A_{L}^{a n} A_{L}^{b n} \rightarrow A_{L}^{c n} A_{L}^{d n}\right]=g^{2}\left(\mathcal{C}_{s} \mathcal{K}_{s}^{\mathrm{el}}+\mathcal{C}_{t} \mathcal{K}_{t}^{\mathrm{el}}+\mathcal{C}_{u} \mathcal{K}_{u}^{\mathrm{el}}\right), \\
& \widetilde{\mathcal{T}}\left[A_{5}^{a n} A_{5}^{b n} \rightarrow A_{5}^{c n} A_{5}^{d n}\right]=g^{2}\left(\mathcal{C}_{s} \widetilde{\mathcal{K}}_{s}^{\mathrm{el}}+\mathcal{C}_{t} \widetilde{\mathcal{K}}_{t}^{\mathrm{el}}+\mathcal{C}_{u} \widetilde{\mathcal{K}}_{u}^{\mathrm{el}}\right),
\end{aligned}
$$

where $\left\{\mathcal{K}_{j}^{\mathrm{el}}\right\}$ denote the kinematic factors for KK gauge bosons,

$$
\begin{aligned}
\mathcal{K}_{s}^{\mathrm{el}} & =-\frac{\left(4 \bar{s}^{2}-5 \bar{s}-8\right) c_{\theta}}{2 \bar{s}}, \\
\mathcal{K}_{t}^{\mathrm{el}} & =-\frac{Q_{0}+Q_{1} c_{\theta}+Q_{2} c_{2 \theta}+Q_{3} c_{3 \theta}}{2(\bar{s}-4)\left[(3 \bar{s}+4)+4 \bar{s} c_{\theta}+(\bar{s}-4) c_{2 \theta}\right]}, \\
\mathcal{K}_{u}^{\mathrm{el}} & =\frac{Q_{0}-Q_{1} c_{\theta}+Q_{2} c_{2 \theta}-Q_{3} c_{3 \theta}}{2(\bar{s}-4)\left[(3 \bar{s}+4)-4 \bar{s} c_{\theta}+(\bar{s}-4) c_{2 \theta}\right]},
\end{aligned}
$$

and $\left\{\widetilde{\mathcal{K}}_{j}^{\text {el }}\right\}$ denote the kinematic factors for KK Goldstone bosons,

$$
\begin{aligned}
\widetilde{\mathcal{K}}_{s}^{\mathrm{el}} & =-\frac{(3 \bar{s}-8) c_{\theta}}{2 \bar{s}}, \\
\widetilde{\mathcal{K}}_{t}^{\mathrm{el}} & =\frac{\widetilde{Q}_{0}+\widetilde{Q}_{1} c_{\theta}+\widetilde{Q}_{2} c_{2 \theta}}{2(\bar{s}-4)\left[(3 \bar{s}+4)+4 \bar{s} c_{\theta}+(\bar{s}-4) c_{2 \theta}\right]}, \\
\widetilde{\mathcal{K}}_{u}^{\mathrm{el}} & =-\frac{\widetilde{Q}_{0}-\widetilde{Q}_{1} c_{\theta}+\widetilde{Q}_{2} c_{2 \theta}}{2(\bar{s}-4)\left[(3 \bar{s}+4)-4 \bar{s} c_{\theta}+(\bar{s}-4) c_{2 \theta}\right]},
\end{aligned}
$$

with the functions $\left\{Q_{j}, \widetilde{Q}_{j}\right\}$ expressed as

$$
\begin{array}{llrl}
Q_{0} & =8 \bar{s}^{3}-63 \bar{s}^{2}+72 \bar{s}+80, & & \widetilde{Q}_{0}=15 \bar{s}^{2}+24 \bar{s}-80, \\
Q_{1} & =2\left(7 \bar{s}^{3}-44 \bar{s}^{2}+80 \bar{s}-64\right), & \widetilde{Q}_{1}=4\left(3 \bar{s}^{2}-20 \bar{s}+32\right), \\
Q_{2}=8 \bar{s}^{3}-45 \bar{s}^{2}+8 \bar{s}+48, & \widetilde{Q}_{2}=-3(\bar{s}-4)^{2}, \\
Q_{3}=2 \bar{s}\left(\bar{s}^{2}-10 \bar{s}+24\right) . & &
\end{array}
$$

Making the high energy expansion of $1 / s$, we can derive the following LO scattering amplitudes:

$$
\begin{array}{rlrl}
\mathcal{K}_{s}^{\mathrm{el} 0} & =\frac{5 c_{\theta}}{2}, & \widetilde{\mathcal{K}}_{s}^{\mathrm{el} 0}=-\frac{3 c_{\theta}}{2}, \\
\mathcal{K}_{t}^{\mathrm{e} 0}=\frac{13+5 c_{\theta}+4 c_{2 \theta}}{2\left(1+c_{\theta}\right)}, & \widetilde{\mathcal{K}}_{t}^{\mathrm{e} 0}=\frac{3\left(3-c_{\theta}\right)}{2\left(1+c_{\theta}\right)}, \\
\mathcal{K}_{u}^{\mathrm{el} 0}=-\frac{13-5 c_{\theta}+4 c_{2 \theta}}{2\left(1-c_{\theta}\right)}, & \widetilde{\mathcal{K}}_{u}^{\mathrm{el} 0}=-\frac{3\left(3+c_{\theta}\right)}{2\left(1-c_{\theta}\right)},
\end{array}
$$

and the NLO scattering amplitudes:

$$
\begin{aligned}
\delta \mathcal{K}_{s}^{\mathrm{el}}=\frac{4 c_{\theta}}{\bar{s}}, & \delta \widetilde{\mathcal{K}}_{s}^{\mathrm{el}}=\frac{4 c_{\theta}}{\bar{s}}, \\
\delta \mathcal{K}_{t}^{\mathrm{el}}=-\frac{8\left(2-3 c_{\theta}-2 c_{2 \theta}-c_{3 \theta}\right)}{\left(3+4 c_{\theta}+c_{2 \theta}\right) \bar{s}}, & \delta \widetilde{\mathcal{K}}_{t}^{\mathrm{el}}=\frac{32 c_{\theta}}{\left(3+4 c_{\theta}+c_{2 \theta}\right) \bar{s}},
\end{aligned}
$$




$$
\delta \mathcal{K}_{u}^{\mathrm{el}}=\frac{8\left(2+3 c_{\theta}-2 c_{2 \theta}+c_{3 \theta}\right)}{\left(3-4 c_{\theta}+c_{2 \theta}\right) \bar{s}}, \quad \delta \widetilde{\mathcal{K}}_{u}^{\mathrm{el}}=\frac{32 c_{\theta}}{\left(3-4 c_{\theta}+c_{2 \theta}\right) \bar{s}} .
$$

Note that the above expansion of $1 / s$ differs from the expansion of $1 / s_{0}$ [cf. eq. (A.3)] as adopted in ref. [32]. We also note that in each channel of $(s, t, u)$ the LO longitudinal KK gauge boson amplitude differs from the LO KK Goldstone boson amplitude by the same amount: $\mathcal{K}_{j}^{\mathrm{el} 0}-\widetilde{\mathcal{K}}_{j}^{\mathrm{el} 0}=4 c_{\theta}$. Hence, due to the Jacobi identity the elastic KK longitudinal gauge boson amplitude and KK Goldstone boson amplitude are equal at the LO, $\mathcal{T}_{0 L}\left[4 A_{L}^{a n}\right]=\widetilde{\mathcal{T}}_{05}\left[4 A_{5}^{a n}\right]$, which verifies the KK gauge boson equivalence theorem (KK GAET) [41][45]. ${ }^{3}$

Then, we further define the BCJ-type numerators:

$$
\begin{array}{ll}
\mathcal{N}_{j}^{\mathrm{el}}=s_{j} \mathcal{K}_{j}^{\mathrm{el}}, & \mathcal{N}_{j}^{\mathrm{el}}=\mathcal{N}_{j}^{\mathrm{el} 0}+\delta \mathcal{N}_{j}^{\mathrm{el}}=s_{j}\left(\mathcal{K}_{j}^{\mathrm{el} 0}+\delta \mathcal{K}_{j}^{\mathrm{el}}\right), \\
\widetilde{\mathcal{N}}_{j}^{\mathrm{el}}=s_{j} \widetilde{\mathcal{K}}_{j}^{\mathrm{el}}, & \widetilde{\mathcal{N}}_{j}^{\mathrm{el}}=\widetilde{\mathcal{N}}_{j}^{\mathrm{el} 0}+\delta \widetilde{\mathcal{N}}_{j}^{\mathrm{el}}=s_{j}\left(\widetilde{\mathcal{K}}_{j}^{\mathrm{el} 0}+\delta \widetilde{\mathcal{K}}_{j}^{\mathrm{el}}\right),
\end{array}
$$

where $j \in(s, t, u)$, and we have decomposed the numerators $\left\{\mathcal{N}_{j}^{\mathrm{el}}, \widetilde{\mathcal{N}}_{j}^{\mathrm{el}}\right\}$ into the LO and NLO parts under high energy expansion. With these, we can reformulate the scattering amplitudes (B.1) as follows:

$$
\begin{aligned}
& \mathcal{T}\left[A_{L}^{a n} A_{L}^{b n} \rightarrow A_{L}^{c n} A_{L}^{d n}\right]=g^{2}\left(\frac{\mathcal{C}_{s} \mathcal{N}_{s}^{\mathrm{el}}}{s}+\frac{\mathcal{C}_{t} \mathcal{N}_{t}^{\mathrm{el}}}{t}+\frac{\mathcal{C}_{u} \mathcal{N}_{u}^{\mathrm{el}}}{u}\right), \\
& \widetilde{\mathcal{T}}\left[A_{5}^{a n} A_{5}^{b n} \rightarrow A_{5}^{c n} A_{5}^{d n}\right]=g^{2}\left(\frac{\mathcal{C}_{s} \widetilde{\mathcal{N}}_{s}^{\mathrm{el}}}{s}+\frac{\mathcal{C}_{t} \widetilde{\mathcal{N}}_{t}^{\mathrm{el}}}{t}+\frac{\mathcal{C}_{u} \widetilde{\mathcal{N}}_{u}^{\mathrm{el}}}{u}\right) .
\end{aligned}
$$

Then, we find that the LO numerators $\left\{\mathcal{N}_{j}^{\mathrm{el} 0}, \widetilde{\mathcal{N}}_{j}^{\mathrm{el} 0}\right\}$ and the NLO numerators $\left\{\delta \mathcal{N}_{j}^{\mathrm{el}}, \delta \widetilde{\mathcal{N}}_{j}^{\mathrm{el}}\right\}$ are both mass-dependent and their sums violate the kinematic Jacobi identity by terms of $\mathcal{O}\left(E^{0} M_{n}^{2}\right)$ and smaller:

$$
\begin{aligned}
& \sum_{j} \mathcal{N}_{j}^{\mathrm{el} 0}=10 c_{\theta} M_{n}^{2}, \quad \sum_{j} \widetilde{\mathcal{N}}_{j}^{\mathrm{el} 0}=-6 c_{\theta} M_{n}^{2}, \\
& \sum_{j} \delta \mathcal{N}_{j}^{\mathrm{el}}=M_{n}^{2}\left[\frac{1}{2}\left(-13+12 c_{2 \theta}+c_{4 \theta}\right)+\frac{8\left(31+c_{4 \theta}\right)}{\bar{s}}\right] c_{\theta} \csc ^{4} \theta \\
& \sum_{j} \delta \widetilde{\mathcal{N}}_{j}^{\mathrm{el}}=M_{n}^{2}\left[\frac{1}{2}\left(-13+12 c_{2 \theta}+c_{4 \theta}\right)+\frac{32\left(7+c_{2 \theta}\right)}{\bar{s}}\right] c_{\theta} \csc ^{4} \theta
\end{aligned}
$$

We note that all the $\mathcal{O}\left(E^{2}\right)$ terms in the LO amplitudes are mass-independent and obey the kinematic Jacobi identity as shown in eq. (B.9a), while all the Jacobi-violating terms in the $\mathrm{LO} / \mathrm{NLO}$ amplitudes are mass-dependent and have $\mathcal{O}\left(E^{0} M_{n}^{2}\right)$ or smaller. Because of these Jacobi-violating terms, the conventional BCJ double-copy method of the massless gauge theories cannot be naively applied to the case of the elastic scattering amplitudes of KK gauge (Goldstone) bosons. However, we note that the amplitudes (B.8) are invariant under the generalized gauge transformations of the kinematic numerators:

$$
\mathcal{N}_{j}^{\mathrm{el} \prime}=\mathcal{N}_{j}^{\mathrm{el}}+s_{j} \Delta^{\mathrm{el}}, \quad \widetilde{\mathcal{N}}_{j}^{\mathrm{el} \prime}=\widetilde{\mathcal{N}}_{j}^{\mathrm{el}}+s_{j} \widetilde{\Delta}^{\mathrm{el}} .
$$

\footnotetext{
${ }^{3}$ The four-point KK gauge boson scattering amplitudes were also computed [54] for the 5d SM under the orbifold compactification of $\mathbb{S}^{1} / \mathbb{Z}_{2}$.
} 
In the above, the gauge parameters $\left(\Delta^{\mathrm{el}}, \widetilde{\Delta}^{\mathrm{el}}\right)$ can be solved by requiring the gaugetransformed numerators to satisfy the Jacobi identities: $\sum_{j} \mathcal{N}_{j}^{\mathrm{el} \prime}=0$ and $\sum_{j} \widetilde{\mathcal{N}}_{j}^{\text {el } \prime}=0$. Thus, we derive the following general solutions:

$$
\Delta^{\mathrm{el}}=-\frac{1}{4 M_{n}^{2}} \sum_{j} \mathcal{N}_{j}^{\mathrm{el}}, \quad \widetilde{\Delta}^{\mathrm{el}}=-\frac{1}{4 M_{n}^{2}} \sum_{j} \widetilde{\mathcal{N}}_{j}^{\mathrm{el}} .
$$

Expanding both sides of eq. (B.11), we derive the gauge parameters $\left(\Delta^{\mathrm{el}}, \widetilde{\Delta}^{\mathrm{el}}\right)=\left(\Delta_{0}^{\mathrm{el}}+\right.$ $\left.\Delta_{1}^{\mathrm{el}}, \widetilde{\Delta}_{0}^{\mathrm{el}}+\widetilde{\Delta}_{1}^{\mathrm{el}}\right)$ at the LO and NLO:

$$
\begin{array}{ll}
\Delta_{0}^{\mathrm{el}}=\frac{1}{4}\left(9+7 c_{2 \theta}\right) c_{\theta} \csc ^{2} \theta, & \widetilde{\Delta}_{0}^{\mathrm{el}}=\frac{1}{4}\left(17-c_{2 \theta}\right) c_{\theta} \csc ^{2} \theta, \\
\Delta_{1}^{\mathrm{el}}=-\bar{s}^{-1} 2\left(31+c_{4 \theta}\right) c_{\theta} \csc ^{4} \theta, & \widetilde{\Delta}_{1}^{\mathrm{el}}=-\bar{s}^{-1} 8\left(7+c_{2 \theta}\right) c_{\theta} \csc ^{4} \theta .
\end{array}
$$

Then, we can extend the conventional BCJ method and apply the color-kinematics duality to the following gauge-transformed scattering amplitudes:

$$
\begin{aligned}
& \mathcal{T}\left[A_{L}^{a n} A_{L}^{b n} \rightarrow A_{L}^{c n} A_{L}^{d n}\right]=g^{2}\left(\frac{\mathcal{C}_{s} \mathcal{N}_{s}^{\mathrm{el} \prime}}{s}+\frac{\mathcal{C}_{t} \mathcal{N}_{t}^{\mathrm{el} \prime}}{t}+\frac{\mathcal{C}_{u} \mathcal{N}_{u}^{\mathrm{el} \prime}}{u}\right) \\
& \widetilde{\mathcal{T}}\left[A_{5}^{a n} A_{5}^{b n} \rightarrow A_{5}^{c n} A_{5}^{d n}\right]=g^{2}\left(\frac{\mathcal{C}_{s} \widetilde{\mathcal{N}}_{s}^{\mathrm{el} \prime}}{s}+\frac{\mathcal{C}_{t} \widetilde{\mathcal{N}}_{t}^{\mathrm{el} \prime}}{t}+\frac{\mathcal{C}_{u} \widetilde{\mathcal{N}}_{u}^{\mathrm{el} \prime}}{u}\right) .
\end{aligned}
$$

We find that this extended BCJ-type double-copy construction gives the correct LO KK graviton (Goldstone) amplitudes at $\mathcal{O}\left(E^{2} M_{n}^{0}\right)$, and also gives the correct structure of the NLO KK graviton (Goldstone) amplitudes at $\mathcal{O}\left(E^{0} M_{n}^{2}\right)$ although the coefficients do not exactly match that of the original KK graviton (Goldstone) amplitudes at the NLO. So, we need proper modifications on the extended BCJ-type double-copy construction of the massive NLO KK gauge/gravity amplitudes, as shown in ref. [32]. In the current study, we have demonstrated in sections 3-4 that the double-copy construction for the massive KK gauge/gravity amplitudes can be successfully realized by using the KK string-based formulation of the extended massive KLT-like relations, which hold for the exact $N$-point tree-level amplitudes without making the high energy expansion.

\section{B.2 Inelastic scattering amplitudes of KK gauge and Goldstone bosons}

In this appendix, we consider the KK YM gauge theory under the 5d compactification of $\mathbb{S}^{1} / \mathbb{Z}_{2}$. We present the full four-point scattering amplitudes for the inelastic channels $(n, n) \rightarrow(m, m)$ and $(0,0) \rightarrow(n, n)$ at tree level, which were not given previously in ref. [32].

\section{B.2.1 Inelastic scattering amplitudes of $(n, n) \rightarrow(m, m)$}

For the inelastic scattering process $(n, n) \rightarrow(m, m)$, we compute the four-point scattering amplitudes of the longitudinal KK gauge and Goldstone bosons:

$$
\begin{aligned}
& \mathcal{T}\left[A_{L}^{a n} A_{L}^{b n} \rightarrow A_{L}^{c m} A_{L}^{d m}\right]=g^{2}\left(\mathcal{C}_{s} \mathcal{K}_{s}^{\mathrm{in}}+\mathcal{C}_{t} \mathcal{K}_{t}^{\mathrm{in}}+\mathcal{C}_{u} \mathcal{K}_{u}^{\mathrm{in}}\right), \\
& \widetilde{\mathcal{T}}\left[A_{5}^{a n} A_{5}^{b n} \rightarrow A_{5}^{c m} A_{5}^{d m}\right]=g^{2}\left(\mathcal{C}_{s} \widetilde{\mathcal{K}}_{s}^{\mathrm{in}}+\mathcal{C}_{t} \widetilde{\mathcal{K}}_{t}^{\mathrm{in}}+\mathcal{C}_{u} \widetilde{\mathcal{K}}_{u}^{\mathrm{in}}\right),
\end{aligned}
$$


where $\left\{\mathcal{K}_{j}^{\text {in }}\right\}$ denote the kinematic factors for KK gauge bosons,

$$
\begin{aligned}
\mathcal{K}_{s}^{\text {in }} & =-\frac{2 \bar{q} \bar{q}^{\prime}\left(\bar{s} r_{+}^{2}+2 r^{2}\right) c_{\theta}}{\bar{s} r^{2}}, \\
\mathcal{K}_{t}^{\text {in }} & =\frac{-\left(Q_{0}+Q_{1} c_{\theta}+Q_{2} c_{2 \theta}+Q_{3} c_{3 \theta}\right)}{r^{2}\left(3 \bar{s}^{2}-4 \bar{s} r_{+}^{2}-16 r^{2}+16 \bar{s} \bar{q} \bar{q}^{\prime} c_{\theta}+4 \bar{q} \bar{q}^{\prime} c_{2 \theta}\right)}, \\
\mathcal{K}_{u}^{\text {in }} & =\frac{Q_{0}-Q_{1} c_{\theta}+Q_{2} c_{2 \theta}-Q_{3} c_{3 \theta}}{r^{2}\left(3 \bar{s}^{2}-4 \bar{s} r_{+}^{2}-16 r^{2}-16 \bar{s} \bar{q} \bar{q}^{\prime} c_{\theta}+4 \bar{q} \bar{q}^{\prime} c_{2 \theta}\right)},
\end{aligned}
$$

and $\left\{\widetilde{\mathcal{K}}_{j}^{\text {in }}\right\}$ denote the kinematic factors for KK Goldstone bosons,

$$
\begin{aligned}
\widetilde{\mathcal{K}}_{s}^{\text {in }} & =-\frac{4 \bar{q} \bar{q}^{\prime} c_{\theta}}{\bar{s}}, \\
\widetilde{\mathcal{K}}_{t}^{\text {in }} & =\frac{\widetilde{Q}_{0}+\widetilde{Q}_{1} c_{\theta}+\widetilde{Q}_{2} c_{2 \theta}}{2\left(\bar{s}^{2}-16 r^{2}+8 \bar{q} \bar{q} \bar{q}^{\prime} c_{\theta}+16 \bar{q}^{2} \bar{q}^{\prime 2} c_{\theta}^{2}\right)}, \\
\widetilde{\mathcal{K}}_{u}^{\text {in }} & =\frac{-\left(\widetilde{Q}_{0}-\widetilde{Q}_{1} c_{\theta}+\widetilde{Q}_{2} c_{2 \theta}\right)}{2\left(\bar{s}^{2}-16 r^{2}-8 \bar{s} \bar{q} \bar{q}^{\prime} c_{\theta}+16 \bar{q}^{2} \bar{q}^{\prime 2} c_{\theta}^{2}\right)},
\end{aligned}
$$

with the polynomial functions $\left\{Q_{j}, \widetilde{Q}_{j}\right\}$ expressed as

$$
\begin{aligned}
& Q_{0}=\bar{s}^{3} r_{+}^{2}-\bar{s}^{2}\left(4 r^{4}+13 r^{2}+4\right)+20 \bar{s} r^{2} r_{+}^{2}-16 r^{4}, \quad \widetilde{Q}_{0}=5 \bar{s}^{2}-16 r^{2}, \\
& Q_{1}=\bar{s} \bar{q} \bar{q}^{\prime}\left[7 \bar{s} r_{+}^{2}-4\left(r_{+}^{2}+2\right)\left(3 r^{2}+1\right)\right], \quad \widetilde{Q}_{1}=16 \bar{q} \bar{q}^{\prime}\left(\bar{s}-r_{+}^{2}\right) \text {, } \\
& Q_{2}=\bar{s}^{3} r_{+}^{2}-\bar{s}^{2}\left(4 r^{4}+7 r^{2}+4\right)+4 \bar{s} r^{2} r_{+}^{2}+16 r^{4}, \quad \widetilde{Q}_{2}=-16 \bar{q}^{2} \bar{q}^{\prime 2}, \\
& Q_{3}=\bar{s} \bar{q} \bar{q}^{\prime} r_{+}^{2}\left(\bar{s}-4 r_{+}^{2}\right) \text {, }
\end{aligned}
$$

where we have introduced the notations $r=M_{m} / M_{n}$ and $r_{+}^{2}=1+r^{2}$, and other kinematic quantities are also defined in eq. (3.11).

Then, we make the high energy expansions for the above scattering amplitudes at the LO and NLO:

$$
\begin{array}{ll}
\mathcal{T}\left[A_{L}^{a n} A_{L}^{b n} \rightarrow A_{L}^{c m} A_{L}^{d m}\right]=\mathcal{T}_{0 L}+\delta \mathcal{T}_{L}, & \widetilde{\mathcal{T}}\left[A_{5}^{a n} A_{5}^{b n} \rightarrow A_{5}^{c m} A_{5}^{d m}\right]=\widetilde{\mathcal{T}}_{05}+\delta \widetilde{\mathcal{T}}_{5}, \\
\mathcal{T}_{0 L}=g^{2}\left(\mathcal{C}_{s} \mathcal{K}_{s}^{\text {in } 0}+\mathcal{C}_{t} \mathcal{K}_{t}^{\text {in } 0}+\mathcal{C}_{u} \mathcal{K}_{u}^{\text {in } 0}\right), & \widetilde{\mathcal{T}}_{05}=g^{2}\left(\mathcal{C}_{s} \widetilde{\mathcal{K}}_{s}^{\text {in } 0}+\mathcal{C}_{t} \widetilde{\mathcal{K}}_{t}^{\text {in } 0}+\mathcal{C}_{u} \widetilde{\mathcal{K}}_{u}^{\text {in } 0}\right), \\
\delta \mathcal{T}_{L}=g^{2}\left(\mathcal{C}_{s} \delta \mathcal{K}_{s}^{\text {in }}+\mathcal{C}_{t} \delta \mathcal{K}_{t}^{\text {in }}+\mathcal{C}_{u} \delta \mathcal{K}_{u}^{\text {in }}\right), & \delta \widetilde{\mathcal{T}}_{5}=g^{2}\left(\mathcal{C}_{s} \delta \widetilde{\mathcal{K}}_{s}^{\text {in }}+\mathcal{C}_{t} \delta \widetilde{\mathcal{K}}_{t}^{\text {in }}+\mathcal{C}_{u} \delta \widetilde{\mathcal{K}}_{u}^{\text {in }}\right) .
\end{array}
$$

We derive the LO inelastic scattering amplitudes as follows:

$$
\begin{array}{ll}
\mathcal{K}_{s}^{\text {in } 0}=c_{\theta}, & \widetilde{\mathcal{K}}_{s}^{\text {in } 0}=-c_{\theta}, \\
\mathcal{K}_{t}^{\text {in } 0}=\frac{4+c_{\theta}+c_{2 \theta}}{1+c_{\theta}}, & \widetilde{\mathcal{K}}_{t}^{\text {in } 0}=\frac{3-c_{\theta}}{1+c_{\theta}}, \\
\mathcal{K}_{u}^{\text {in } 0}=-\frac{4-c_{\theta}+c_{2 \theta}}{1-c_{\theta}}, & \widetilde{\mathcal{K}}_{u}^{\text {in } 0}=-\frac{3+c_{\theta}}{1-c_{\theta}},
\end{array}
$$

where we have removed a common mass-dependent term $\left(r^{2}+r^{-2}\right) c_{\theta}$ in each $\mathcal{K}_{j}^{\text {in } 0}$ by using the Jacobi identity $\mathcal{C}_{s}+\mathcal{C}_{t}+\mathcal{C}_{u}=0$. Thus, the remaining full LO amplitude is still 
mass-independent, as we would expect. Furthermore, we find that in each channel of $(s, t, u)$ the longitudinal KK gauge boson amplitude and KK Goldstone boson amplitude differ by the same amount at the LO (which is $r$-independent):

$$
\mathcal{K}_{s}^{\text {in } 0}-\widetilde{\mathcal{K}}_{s}^{\text {in } 0}=\mathcal{K}_{t}^{\text {in } 0}-\widetilde{\mathcal{K}}_{t}^{\text {in } 0}=\mathcal{K}_{u}^{\text {in } 0}-\widetilde{\mathcal{K}}_{u}^{\text {in } 0}=2 c_{\theta} .
$$

Hence, due to the Jacobi identity the inelastic longitudinal KK gauge and Goldstone boson amplitudes are equal, $\mathcal{T}_{0 L}\left[A_{L}^{a n / m}\right]=\widetilde{\mathcal{T}}_{05}\left[A_{5}^{a n / m}\right]$, in accord with the KK gauge boson equivalence theorem (KK GAET) [41, 45].

We further compute the inelastic scattering amplitudes at the NLO and derive these scattering amplitudes as follows:

$$
\begin{aligned}
\delta \mathcal{K}_{s}^{\text {in }} & =\frac{r_{+}^{2}\left(1+r^{4}\right) c_{\theta}}{r^{2} \bar{s}}, & \delta \widetilde{\mathcal{K}}_{s}^{\text {in }} & =\frac{2 r_{+}^{2} c_{\theta}}{\bar{s}}, \\
\delta \mathcal{K}_{t}^{\text {in }} & =\frac{r_{+}^{2}\left(R_{0}+R_{1} c_{\theta}+R_{2} c_{2 \theta}+R_{3} c_{3 \theta}\right)}{2 r^{2}\left(3+4 c_{\theta}+c_{2 \theta}\right) \bar{s}}, & \delta \widetilde{\mathcal{K}}_{t}^{\text {in }} & =-\frac{4 r_{+}^{2}\left(1-3 c_{\theta}\right)}{\left(3+4 c_{\theta}+c_{2 \theta}\right) \bar{s}}, \\
\delta \mathcal{K}_{u}^{\text {in }} & =-\frac{r_{+}^{2}\left(R_{0}-R_{1} c_{\theta}+R_{2} c_{2 \theta}-R_{3} c_{3 \theta}\right)}{2 r^{2}\left(3-4 c_{\theta}+c_{2 \theta}\right) \bar{s}}, & \delta \widetilde{\mathcal{K}}_{u}^{\text {in }} & =\frac{4 r_{+}^{2}\left(1+3 c_{\theta}\right)}{\left(3-4 c_{\theta}+c_{2 \theta}\right) \bar{s}},
\end{aligned}
$$

where we have adopted the following notations:

$$
\begin{aligned}
R_{0} & =4\left(1-6 r^{2}+r^{4}\right), & & R_{1}=7+10 r^{2}+7 r^{4}, \\
R_{2} & =4\left(1+r^{2}\right)^{2}, & & R_{3}=1+6 r^{2}+r^{4}, \\
r & =M_{m} / M_{n}, & & r_{+}^{2}=1+r^{2} .
\end{aligned}
$$

Then, we define the following LO and NLO inelastic numerators:

$$
\begin{array}{ll}
\mathcal{N}_{j}^{\text {in } 0}=s_{j} \mathcal{K}_{j}^{\text {in } 0}, & \delta \mathcal{N}_{j}^{\text {in } 0}=s_{j} \delta \mathcal{K}_{j}^{\text {in } 0}, \\
\widetilde{\mathcal{N}}_{j}^{\text {in } 0}=s_{j} \widetilde{\mathcal{K}}_{j}^{\text {in } 0}, & \delta \widetilde{\mathcal{N}}_{j}^{\text {in } 0}=s_{j} \delta \widetilde{\mathcal{K}}_{j}^{\text {in } 0} .
\end{array}
$$

Thus, we compute their sums at the LO and NLO. We find that the sums of these numerators violate the kinematic Jacobi identities, $\sum_{j} \mathcal{N}_{j}^{\text {in }} \neq 0$ and $\sum_{j} \widetilde{\mathcal{N}}_{j}^{\text {in }} \neq 0$. Thus, to recover the kinematic Jacobi identity, we make the following generalized gauge transformations for the inelastic numerators:

$$
\mathcal{N}_{j}^{\text {in }}=\mathcal{N}_{j}^{\text {in }}+s_{j} \Delta^{\text {in }}, \quad \widetilde{\mathcal{N}}_{j}^{\text {in }}=\widetilde{\mathcal{N}}_{j}^{\text {in }}+s_{j} \widetilde{\Delta}^{\text {in }},
$$

under which the scattering amplitudes (B.14a)-(B.14b) are invariant. Then, imposing the kinematic Jacobi identities on the gauge-transformed amplitudes $\sum_{j} \mathcal{N}_{j}^{\text {in' }}=0$ and $\sum_{j} \widetilde{\mathcal{N}}_{j}^{\text {in'}}=0$, we derive the general solutions of the gauge parameters $\left(\Delta^{\text {in }}, \widetilde{\Delta}^{\text {in }}\right)$ as follows:

$$
\Delta^{\text {in }}=-\frac{1}{2\left(M_{n}^{2}+M_{m}^{2}\right)} \sum_{j} \mathcal{N}_{j}^{\text {in }}, \quad \widetilde{\Delta}^{\text {in }}=-\frac{1}{2\left(M_{n}^{2}+M_{m}^{2}\right)} \sum_{j} \widetilde{\mathcal{N}}_{j}^{\text {in }} .
$$

For the simplicity of illustration, we set $r=2$ and compute explicitly the sums of the inelastic numerators to the $\mathcal{O}\left(E^{-2}\right)$ :

$$
\begin{aligned}
& \sum_{j} \mathcal{N}_{j}^{\text {in }}=-\frac{1}{4} M_{n}^{2} r_{+}^{2}\left[\left(7+25 c_{2 \theta}\right)-\frac{\left(20867 c_{\theta}-900 c_{2 \theta}+1025 c_{4 \theta}\right)}{20 s_{\theta}^{2} \bar{s}}\right] c_{\theta} \csc ^{2} \theta, \\
& \sum_{j} \widetilde{\mathcal{N}}_{j}^{\text {in }}=-8 M_{n}^{2} r_{+}^{2}\left[1-\frac{\left(565+91 c_{2 \theta}\right)}{20 s_{\theta}^{2} \bar{s}}\right] c_{\theta} \csc ^{2} \theta .
\end{aligned}
$$


where $r=M_{m} / M_{n}$ and $r_{+}^{2}=1+r^{2}$. With the above, we make high energy expansion for the general solutions (B.25) and derive explicitly the LO and NLO of gauge parameters:

$$
\begin{aligned}
& \Delta_{0}^{\text {in }}=\frac{1}{8}\left(7+25 c_{2 \theta}\right) c_{\theta} \csc ^{2} \theta, \\
& \widetilde{\Delta}_{0}^{\text {in }}=4 c_{\theta} \csc ^{2} \theta, \\
& \Delta_{1}^{\text {in }}=-\frac{\left(20867 c_{\theta}-900 c_{2 \theta}+1025 c_{4 \theta}\right) c_{\theta} \csc ^{4} \theta}{160 \bar{s}}, \\
& \widetilde{\Delta}_{1}^{\text {in }}=-\frac{\left(565+91 c_{2 \theta}\right) c_{\theta} \csc ^{4} \theta}{5 \bar{s}},
\end{aligned}
$$

where we have set $r=2$ for illustration.

\section{B.2.2 Inelastic scattering amplitudes of $(0,0) \rightarrow(n, n)$}

Next, we study another inelastic channel $(0,0) \rightarrow(n, n)$. We compute the following full tree-level scattering amplitudes of KK gauge bosons and of KK Goldstone bosons:

$$
\begin{aligned}
& \mathcal{T}\left[A_{ \pm 1}^{0} A_{\mp 1}^{0} \rightarrow A_{L}^{n} A_{L}^{n}\right]=g^{2}\left(\mathcal{C}_{s} \mathcal{K}_{s}^{\text {in }}+\mathcal{C}_{t} \mathcal{K}_{t}^{\text {in }}+\mathcal{C}_{u} \mathcal{K}_{u}^{\text {in }}\right), \\
& \widetilde{\mathcal{T}}\left[A_{ \pm 1}^{0} A_{\mp 1}^{0} \rightarrow A_{5}^{n} A_{5}^{n}\right]=g^{2}\left(\mathcal{C}_{s} \widetilde{\mathcal{K}}_{s}^{\text {in }}+\mathcal{C}_{t} \widetilde{\mathcal{K}}_{t}^{\text {in }}+\mathcal{C}_{u} \widetilde{\mathcal{K}}_{u}^{\text {in }}\right),
\end{aligned}
$$

where the sub-amplitudes $\left\{\mathcal{K}_{j}^{\text {in }}\right\}$ and $\left\{\widetilde{\mathcal{K}}_{j}^{\text {in }}\right\}$ are given by

$$
\begin{array}{rlrl}
\mathcal{K}_{s}^{\text {in }} & =0, & \widetilde{\mathcal{K}}_{s}^{\text {in }}=0, \\
\mathcal{K}_{t}^{\text {in }}=\frac{-(\bar{s}+4) s_{\theta}^{2}}{\bar{s}+[\bar{s}(\bar{s}-4)]^{1 / 2} c_{\theta}}, & \widetilde{\mathcal{K}}_{t}^{\text {in }}=\frac{-(\bar{s}-4) s_{\theta}^{2}}{\bar{s}+[\bar{s}(\bar{s}-4)]^{1 / 2} c_{\theta}}, \\
\mathcal{K}_{u}^{\text {in }}=\frac{(\bar{s}+4) s_{\theta}^{2}}{\bar{s}-[\bar{s}(\bar{s}-4)]^{1 / 2} c_{\theta}}, & \widetilde{\mathcal{K}}_{u}^{\text {in }}=\frac{(\bar{s}-4) s_{\theta}^{2}}{\bar{s}-[\bar{s}(\bar{s}-4)]^{1 / 2} c_{\theta}} .
\end{array}
$$

Then, we make the high energy expansions of the above scattering amplitudes at the LO and NLO:

$$
\begin{array}{ll}
\mathcal{T}\left[A_{ \pm 1}^{0} A_{\mp 1}^{0} \rightarrow A_{L}^{n} A_{L}^{n}\right]=\mathcal{T}_{0 L}+\delta \mathcal{T}_{L}, & \widetilde{\mathcal{T}}\left[A_{ \pm 1}^{0} A_{\mp 1}^{0} \rightarrow A_{5}^{n} A_{5}^{n}\right]=\widetilde{\mathcal{T}}_{05}+\delta \widetilde{\mathcal{T}}_{5}, \\
\mathcal{T}_{0 L}=g^{2}\left(\mathcal{C}_{t} \mathcal{K}_{t}^{\text {in } 0}+\mathcal{C}_{u} \mathcal{K}_{u}^{\text {in } 0}\right), & \widetilde{\mathcal{T}}_{05}=g^{2}\left(\mathcal{C}_{t} \widetilde{\mathcal{K}}_{t}^{\text {in } 0}+\mathcal{C}_{u} \widetilde{\mathcal{K}}_{u}^{\text {in } 0}\right), \\
\delta \mathcal{T}_{L}=g^{2}\left(\mathcal{C}_{t} \delta \mathcal{K}_{t}^{\text {in }}+\mathcal{C}_{u} \delta \mathcal{K}_{u}^{\text {in }}\right), & \delta \widetilde{\mathcal{T}}_{5}=g^{2}\left(\mathcal{C}_{t} \delta \widetilde{\mathcal{K}}_{t}^{\text {in }}+\mathcal{C}_{u} \delta \widetilde{\mathcal{K}}_{u}^{\text {in }}\right) .
\end{array}
$$

Since the $s$-channel sub-amplitudes vanish, $\mathcal{K}_{s}^{\text {in }}=\widetilde{\mathcal{K}}_{s}^{\text {in }}=0$, we derive the following LO inelastic sub-amplitudes for $(t, u)$ channels,

$$
\mathcal{K}_{t}^{\text {in } 0}=\widetilde{\mathcal{K}}_{t}^{\text {in } 0}=-\left(1-c_{\theta}\right), \quad \mathcal{K}_{u}^{\text {in } 0}=\widetilde{\mathcal{K}}_{u}^{\text {in } 0}=1+c_{\theta},
$$

and the following NLO inelastic sub-amplitudes,

$$
\begin{array}{rlrl}
\delta \mathcal{K}_{t}^{\text {in }} & =-\frac{1+2 c_{\theta}-3 c_{2 \theta}}{\left(1+c_{\theta}\right) \bar{s}}, & \delta \widetilde{\mathcal{K}}_{t}^{\text {in }} & =\frac{3-2 c_{\theta}-c_{2 \theta}}{\left(1+c_{\theta}\right) \bar{s}}, \\
\delta \mathcal{K}_{u}^{\text {in }}=\frac{1-2 c_{\theta}-3 c_{2 \theta}}{\left(1-c_{\theta}\right) \bar{s}}, & \delta \widetilde{\mathcal{K}}_{u}^{\text {in }} & =-\frac{3+2 c_{\theta}-c_{2 \theta}}{\left(1-c_{\theta}\right) \bar{s}} .
\end{array}
$$


Then, we define the LO and NLO numerators of the inelastic amplitude as in eq. (B.23). With these, we compute the sums of the inelastic numerators to the $\mathcal{O}\left(E^{-2}\right)$ :

$$
\begin{aligned}
& \sum_{j} \mathcal{N}_{j}^{\text {in }}=2 c_{\theta} M_{n}^{2}\left[1-\frac{\left(1+3 c_{2 \theta}\right) \csc ^{2} \theta}{\bar{s}}\right], \\
& \sum_{j} \widetilde{\mathcal{N}}_{j}^{\text {in }}=2 c_{\theta} M_{n}^{2}\left[1-\frac{\left(5-c_{2 \theta}\right) \csc ^{2} \theta}{\bar{s}}\right] .
\end{aligned}
$$

To recover the kinematic Jacobi identities, we make the generalized gaugetransformations (B.24) on the numerators such that $\sum_{j} \mathcal{N}_{j}^{\text {in' }}=0$ and $\sum_{j} \widetilde{\mathcal{N}}_{j}^{\text {in' }}=0$. Thus, we derive the following general solutions of the gauge parameters $\left(\Delta^{\text {in }}, \widetilde{\Delta}^{\text {in }}\right)$ :

$$
\Delta^{\text {in }}=-\frac{1}{2 M_{n}^{2}} \sum_{j} \mathcal{N}_{j}^{\text {in }}, \quad \widetilde{\Delta}^{\text {in }}=-\frac{1}{2 M_{n}^{2}} \sum_{j} \widetilde{\mathcal{N}}_{j}^{\text {in }} .
$$

Finally, under high energy expansion, we can derive the LO and NLO gauge parameters as follows:

$$
\Delta_{0}^{\text {in }}=\widetilde{\Delta}_{0}^{\text {in }}=-c_{\theta}, \quad \Delta_{1}^{\text {in }}=\frac{\left(1+3 c_{2 \theta}\right) c_{\theta} \csc ^{2} \theta}{\bar{s}}, \quad \widetilde{\Delta}_{1}^{\text {in }}=\frac{\left(5-c_{2 \theta}\right) c_{\theta} \csc ^{2} \theta}{\bar{s}} .
$$

Open Access. This article is distributed under the terms of the Creative Commons Attribution License (CC-BY 4.0), which permits any use, distribution and reproduction in any medium, provided the original author(s) and source are credited.

\section{References}

[1] T. Kaluza, Zum Unitätsproblem der Physik, Sitzungsber. Preuss. Akad. Wiss. Berlin (Math. Phys. ) 1921 (1921) 966 [arXiv:1803.08616] [INSPIRE].

[2] O. Klein, Quantum theory and five-dimensional theory of relativity, Z. Phys. 37 (1926) 895 [INSPIRE].

[3] M.B. Green, J.H. Schwarz and E. Witten, Superstring theory, Cambridge University Press, Cambricge U.K. (1987).

[4] J. Polchinski, String theory, Cambridge University Press, Cambridge U.K. (1998).

[5] I. Antoniadis, A possible new dimension at a few TeV, Phys. Lett. B 246 (1990) 377 [INSPIRE].

[6] N. Arkani-Hamed, S. Dimopoulos and G.R. Dvali, The hierarchy problem and new dimensions at a millimeter, Phys. Lett. B 429 (1998) 263 [hep-ph/9803315] [INSPIRE].

[7] I. Antoniadis, N. Arkani-Hamed, S. Dimopoulos and G.R. Dvali, New dimensions at a millimeter to a Fermi and superstrings at a TeV, Phys. Lett. B 436 (1998) 257 [hep-ph/9804398] [INSPIRE].

[8] L. Randall and R. Sundrum, A large mass hierarchy from a small extra dimension, Phys. Rev. Lett. 83 (1999) 3370 [hep-ph/9905221] [INSPIRE].

[9] L. Randall and R. Sundrum, An alternative to compactification, Phys. Rev. Lett. 83 (1999) 4690 [hep-th/9906064] [INSPIRE]. 
[10] S.L. Glashow, Partial symmetries of weak interactions, Nucl. Phys. 22 (1961) 579 [InSPIRE].

[11] S. Weinberg, A model of leptons, Phys. Rev. Lett. 19 (1967) 1264 [INSPIRE].

[12] A. Salam, Weak and Electromagnetic Interactions, in Elementary particle theory, Nobel symposium, N. Svartholm ed., Almqvist \& Wiksells, Stockholm Sweden (1968).

[13] H. Georgi and S.L. Glashow, Unity of all elementary particle forces, Phys. Rev. Lett. 32 (1974) 438 [INSPIRE].

[14] H. Georgi, H.R. Quinn and S. Weinberg, Hierarchy of interactions in unified gauge theories, Phys. Rev. Lett. 33 (1974) 451 [INSPIRE].

[15] S. Dimopoulos and H. Georgi, Softly broken supersymmetry and SU(5), Nucl. Phys. B 193 (1981) 150 [INSPIRE].

[16] S. Dimopoulos, S. Raby and F. Wilczek, Supersymmetry and the scale of unification, Phys. Rev. D 24 (1981) 1681 [INSPIRE].

[17] H. Kawai, D.C. Lewellen and S.H.H. Tye, A relation between tree amplitudes of closed and open strings, Nucl. Phys. B 269 (1986) 1 [INSPIRE].

[18] Z. Bern, J.J.M. Carrasco and H. Johansson, New relations for gauge-theory amplitudes, Phys. Rev. D 78 (2008) 085011 [arXiv: 0805.3993] [INSPIRE].

[19] Z. Bern, J.J.M. Carrasco and H. Johansson, Perturbative quantum gravity as a double copy of gauge theory, Phys. Rev. Lett. 105 (2010) 061602 [arXiv:1004.0476] [INSPIRE].

[20] Z. Bern, J.J. Carrasco, M. Chiodaroli, H. Johansson and R. Roiban, The duality between color and kinematics and its applications, arXiv:1909.01358 [INSPIRE].

[21] S.H. Henry Tye and Y. Zhang, Dual identities inside the gluon and the graviton scattering amplitudes, JHEP 06 (2010) 071 [Erratum ibid. 04 (2011) 114] [arXiv: 1003.1732] [INSPIRE].

[22] F. Cachazo, S. He and E.Y. Yuan, Scattering equations and Kawai-Lewellen-Tye orthogonality, Phys. Rev. D 90 (2014) 065001 [arXiv:1306.6575] [InSPIRE].

[23] F. Cachazo, S. He and E.Y. Yuan, Scattering of massless particles in arbitrary dimensions, Phys. Rev. Lett. 113 (2014) 171601 [arXiv:1307.2199] [INSPIRE].

[24] F. Cachazo, S. He and E.Y. Yuan, Scattering of massless particles: scalars, gluons and gravitons, JHEP 07 (2014) 033 [arXiv:1309.0885] [INSPIRE].

[25] F. Cachazo, S. He and E.Y. Yuan, Einstein-Yang-Mills scattering amplitudes from scattering equations, JHEP 01 (2015) 121 [arXiv:1409.8256] [INSPIRE].

[26] F. Cachazo, S. He and E.Y. Yuan, Scattering equations and matrices: from einstein to Yang-Mills, DBI and NLSM, JHEP 07 (2015) 149 [arXiv:1412.3479] [INSPIRE].

[27] C. de Rham and G. Gabadadze, Generalization of the Fierz-Pauli action, Phys. Rev. D 82 (2010) 044020 [arXiv: 1007.0443] [INSPIRE].

[28] C. de Rham, G. Gabadadze and A.J. Tolley, Resummation of massive gravity, Phys. Rev. Lett. 106 (2011) 231101 [arXiv: 1011.1232] [INSPIRE].

[29] A. Momeni, J. Rumbutis and A.J. Tolley, Massive gravity from double copy, JHEP 12 (2020) 030 [arXiv: 2004.07853] [INSPIRE].

[30] L.A. Johnson, C.R.T. Jones and S. Paranjape, Constraints on a massive double-copy and applications to massive gravity, JHEP 02 (2021) 148 [arXiv:2004.12948] [INSPIRE]. 
[31] A. Momeni, J. Rumbutis and A.J. Tolley, Kaluza-Klein from colour-kinematics duality for massive fields, JHEP 08 (2021) 081 [arXiv: 2012.09711] [INSPIRE].

[32] Y.-F. Hang and H.-J. He, Structure of Kaluza-Klein graviton scattering amplitudes from gravitational equivalence theorem and double-copy, Phys. Rev. D (2022), in Press, [arXiv:2106.04568] [INSPIRE].

[33] A. Agarwal, N. Beisert and T. McLoughlin, Scattering in mass-deformed $N \geq 4$ Chern-Simons models, JHEP 06 (2009) 045 [arXiv:0812.3367] [INSPIRE].

[34] T. Bargheer, S. He and T. McLoughlin, New relations for three-dimensional supersymmetric scattering amplitudes, Phys. Rev. Lett. 108 (2012) 231601 [arXiv:1203.0562] [INSPIRE].

[35] Y.-t. Huang and H. Johansson, Equivalent $D=3$ supergravity amplitudes from double copies of three-algebra and two-algebra gauge theories, Phys. Rev. Lett. 110 (2013) 171601 [arXiv: 1210.2255] [INSPIRE].

[36] N. Moynihan, Scattering amplitudes and the double copy in topologically massive theories, JHEP 12 (2020) 163 [arXiv:2006.15957] [INSPIRE].

[37] D.J. Burger, W.T. Emond and N. Moynihan, Anyons and the double copy, JHEP 01 (2022) 017 [arXiv: 2103.10416] [INSPIRE].

[38] M.C. González, A. Momeni and J. Rumbutis, Massive double copy in three spacetime dimensions, JHEP 08 (2021) 116 [arXiv:2107.00611] [INSPIRE].

[39] N. Moynihan, Massive covariant colour-kinematics in 3D, arXiv:2110.02209 [INSPIRE].

[40] Y.-F. Hang, H.-J. He, and C. Shen, Structure of Chern-Simons scattering amplitudes from topological equivalence theorem and double-copy, JHEP 01 (2022) 153 [arXiv:2110.05399] [INSPIRE].

[41] R.S. Chivukula, D.A. Dicus, and H.-J. He, Unitarity of compactified five-dimensional Yang-Mills theory, Phys. Lett. B 525 (2002) 175 [hep-ph/0111016] [INSPIRE].

[42] L. Dolan and M.J. Duff, Kac-Moody symmetries of Kaluza-Klein theories, Phys. Rev. Lett. 52 (1984) 14 [INSPIRE].

[43] Y.M. Cho and S.W. Zoh, Explicit construction of massive spin two fields in Kaluza-Klein theory, Phys. Rev. D 46 (1992) R2290 [inSPIRE].

[44] R.S. Chivukula and H.-J. He, Unitarity of deconstructed five-dimensional Yang-Mills theory, Phys. Lett. B 532 (2002) 121 [hep-ph/0201164] [INSPIRE].

[45] H.-J. He, Higgsless deconstruction without boundary condition, Int. J. Mod. Phys. A 20 (2005) 3362 [hep-ph/0412113] [INSPIRE].

[46] S. Deser, R. Jackiw and S. Templeton, Three-dimensional massive gauge theories, Phys. Rev. Lett. 48 (1982) 975 [INSPIRE].

[47] S. Deser, R. Jackiw and S. Templeton, Topologically massive gauge theories, Annals Phys. 140 (1982) 372 [Erratum ibid. 185 (1988) 406] [INSPIRE].

[48] J. Gomis, Z. Yan and M. Yu, KLT factorization of winding string amplitudes, JHEP 06 (2021) 057 [arXiv: 2103.05013] [INSPIRE].

[49] R.S. Chivukula, D. Foren, K.A. Mohan, D. Sengupta and E.H. Simmons, Scattering amplitudes of massive spin-2 Kaluza-Klein states grow only as $\mathcal{O}(s)$, Phys. Rev. D 101 (2020) 055013 [arXiv: 1906.11098] [INSPIRE]. 
[50] R.S. Chivukula, D. Foren, K.A. Mohan, D. Sengupta and E.H. Simmons, Massive spin-2 scattering amplitudes in extra-dimensional theories, Phys. Rev. D 101 (2020) 075013 [arXiv: 2002.12458] [INSPIRE].

[51] Z. Bern, L.J. Dixon, M. Perelstein and J.S. Rozowsky, Multileg one loop gravity amplitudes from gauge theory, Nucl. Phys. B 546 (1999) 423 [hep-th/9811140] [INSPIRE].

[52] N.E.J. Bjerrum-Bohr, P.H. Damgaard, T. Sondergaard and P. Vanhove, The momentum kernel of gauge and gravity theories, JHEP 01 (2011) 001 [arXiv:1010.3933] [INSPIRE].

[53] P.D. Francesco, P. Mathieu and D. Senechal, Conformal field theory, Springer, Germany (1997).

[54] R. S. Chivukula, D. A. Dicus, H.-J. He, and S. Nandi, Unitarity of the higher dimensional standard model, Phys. Lett. B 562 (2003) 109 [hep-ph/0302263] [INSPIRE]. 